\title{
Spatially Explicit Spectral Analysis of Point Clouds and Geospatial Data.
}

\author{
Daniel Buscombe* \\ Grand Canyon Monitoring and Research Center, Southwest Biological Science Center, U.S. Geological \\ Survey, Flagstaff, Arizona, USA
}

\begin{abstract}
The increasing use of spatially explicit analyses of high-resolution spatially distributed data (imagery and point clouds) for the purposes of characterising spatial heterogeneity in geophysical phenomena necessitates the development of custom analytical and computational tools. In recent years, such analyses have become the basis of, for example, automated texture characterisation and segmentation, roughness and grain size calculation, and feature detection and classification, from a variety of data types. In this work, much use has been made of statistical descriptors of localised spatial variations in amplitude variance (roughness), however the horizontal scale (wavelength) and spacing of roughness elements is rarely considered. This is despite the fact that the ratio of characteristic vertical to horizontal scales is not constant and can yield important information about physical scaling relationships. Spectral analysis is a hitherto under-utilised but powerful means to acquire statistical information about relevant amplitude and wavelength scales, simultaneously and with computational efficiency. Further, quantifying spatially distributed data in the frequency domain lends itself to the development of stochastic models for probing the underlying mechanisms which govern the spatial distribution of geological and geophysical phenomena. The software package PySESA (Python program for Spatially Explicit Spectral Analysis) has been developed for generic analyses of spatially distributed data in both the spatial and frequency domains. Developed predominantly in Python, it accesses libraries written in Cython and C++ for efficiency. It is open source and modular, therefore readily incorporated into, and combined with, other data analysis tools and frameworks with particular utility for supporting research in the fields of geomorphology, geophysics, hydrography, photogrammetry and remote sensing. The analytical and computational structure of the toolbox is described, and its functionality illustrated with an example of a high-resolution bathymetric point cloud data collected with multibeam echosounder.
\end{abstract}

Keywords: point cloud, spectral analysis, geospatial analysis, roughness, texture, remote sensing

\footnotetext{
* Corresponding author

Email address: dbuscombe@usgs.gov (Daniel Buscombe)
} 


\section{Introduction}

\subsection{The growing use of high-resolution point clouds in the geosciences}

Across a broad range of geoscience disciplines, interrogating the information in highresolution spatially distributed data (point clouds) for the purposes of, for example, facies description and grain size calculation (e.g. Hodge et al., 2009; Nelson et al., 2014), geomorphic feature detection and classification (e.g. Burrough et al., 2000; Glenn et al., 2006; Pirotti and Tarolli, 2010), vegetation structure description (e.g. Antonarakis et al., 2009; Dassot et al., 2011), and physical habitat quantification (e.g. Vierling et al., 2008; Wheaton et al., 2010; Lassueur et al., 2006; Pradervand et al., 2014) has become increasingly widespread. The increasing accessibility and use of high-resolution topographic point clouds obtained using Light Detection and Ranging (LiDAR) (e.g. Buckley et al., 2008; Hilldale and Raff, 2008), Structure from Motion (SfM) photogrammetry (e.g. James and Robson, 2012; Westoby et al., 2012; Fonstad et al., 2013; Woodget et al., 2015), and range imaging (e.g. Nitsche et al., 2013) has found widespread application in geomorphology (Roering et al., 2013; Tarolli, 2014). The use of singlebeam and multibeam echosounders for bathymetric point cloud collection is on the ascendancy (Mayer, 2006) in geophysical and geomorphological research, and is becoming viable in increasingly shallow water (e.g. Parsons et al., 2005; Wright and Kaplinski, 2011; Buscombe et al., 2014b).

\subsection{Spatially explicit analysis of topographic point clouds}

With these technological developments, the heights of natural surfaces can now be measured with such spatial density that almost the entire spectrum of physical roughness scales can be characterised, down to the form and even grain scales (Brasington et al., 2012). Such 'microtopography' has created a demand for analytical and computational tools for spatially explicit (also known as spatially distributed) statistical characterization of the data (e.g. Keller et al., 1987; Church, 1988; Shepard et al., 2001; Manes et al., 2008; Pollyea and Fairley, 2011, 2012; Rychkov et al., 2012; Brasington et al., 2012; Trevisani et al., 2012; Kukko et al., 2013; Buscombe et al., 2014a). The basic premise is that the point cloud captures a surface whose statistical properties vary in space. Analysing data within small moving windows, calculating relevant statistics and spatially referencing them so they are represented in a decimated point cloud form, captures the spatial variability in the data and allows continuous mapping of statistical quantities such as roughness. This approach has found numerous applications in characterising rough surfaces (Smith, 2014). Of particular interest in roughness characterization is the extreme values, the width of the height distribution, or the length of the distribution tails. As such, the use of the root-mean-square (RMS) or standard deviation of heights (e.g. Shepard et al., 2001; Sankey et al., 2010; Nield et al., 2011) or amplitudes relative to a plane (Shepard et al., 2001; Frankel and Dolan, 2007; Pollyea and Fairley, 2011; Brasington et al., 2012) have become popular means to quantify surface roughness.

\subsection{A case for appropriate scaling of terrestrial roughness statistics}

The variance in amplitudes of a great many of geophysical quantities, including terrestrial surface heights, as a function of wavelength usually obeys a power law (Sayles and Thomas, 1978; Turcotte, 1992). An important consequence of power-law behaviour 
is that RMS roughness, however defined, is scale-dependent (Sayles and Thomas, 1978; Jackson and Richardson, 2007) and insufficient to discriminate between surfaces with multiple roughness length scales. Despite this, the horizontal scale and spacing of roughness elements is rarely considered (Smith, 2014) therefore the amplitude roughness is rarely scaled by the horizontal spacing of amplitude deviations. The ratio of vertical (e.g. standard deviation of heights) to horizontal (e.g. characteristic wavelength) scales is rarely constant (Furbish, 1987). This suggests that the shape, orientation, inclination, spacing and clustering of roughness 'elements' is important, as well as their vertical amplitude (Nikora et al., 1998; Pollyea and Fairley, 2012). These (non-amplitude) factors give vital context to a given surface such as a streambed, seafloor, deflation surface, outcrop or till fabric. In the terminology of fractals, rough surfaces are therefore called 'self-affine' because a different scaling — called a Hurst number or Hausdorff exponent -is required in the horizontal than in the vertical for them both to scale with each other (Turcotte, 1992; Wilson and Dominic, 1998). A small Hurst number, for example, indicates that a surface smooths disproportionately with increasing lengthscale (the surface is rough up close and appears smooth at a distance). It is unlikely that terrestrial surfaces can be reliably distinguished from each other based on these scaling relationships alone (Shepard et al., 2001). Measures of roughness are more physically meaningful if expressed as a parameter which scales vertical roughness to horizontal length characteristic scales. In the geomorphologic sense, if 'roughness' is a measure of the statistical variation in the distribution of topographic relief of a surface, then 'texture' can be defined as the frequency of change and arrangement of roughness.

\subsection{Spatial explicit spectral analysis of point clouds}

Perhaps the most efficient and widespread means with which to simultaneously quantify multi-scalar amplitudes and wavelengths in spatially distributed data, thereby simultaneously quantifying roughness and texture at multiple scales, is through application of spectral analyses (e.g. Fara and Scheidegger, 1961; Gilman et al., 1963; Sayles and Thomas, 1978; Hough, 1989; Perron et al., 2008; Hani et al., 2011; Trevisani et al., 2012). Results of spectral analyses have the additional benefit of being amenable to theoretical stochastic models of surface roughness, especially those that relate surface characteristics to the scattering of light (Miller and Parsons, 1990; Whitehouse, 1997), radar (van Zyl et al., 1991; Shepard et al., 1995) and sound (Jackson and Richardson, 2007).

Spectral analyses of spatially distributed data have proved beneficial for a number of geophysical fields, including characterizing evolving topography (e.g. Cataño-Lopera et al., 2009; Aberle et al., 2010; Singh et al., 2012), topographic feature extraction (e.g. Lashermes et al., 2007; Booth et al., 2009; Passalacqua et al., 2010; Kalbermatten et al., 2012; Berti et al., 2013), grain size analysis (Buscombe and Rubin, 2012; Buscombe, 2013) and, classically, scaling and roughness of terrains (e.g. Rozema, 1968; Pike and Wesley, 1975; Rothrock and Thorndike, 1980; Fox and Hayes, 1985; Family, 1986; Balmino, 1993). Spatially explicit analysis of lengthscales in data can also inform appropriate spatial density of sampling (Pelgrum et al., 2000). Yet, in the catalogue of computational analytical tools now available to analyse the multiscale structures of geophysical, geomorphological and remote sensing point cloud data, conspicuous in its absence are accessible, open-source and generalised computational tools to describe the spatial continuity of the fields they represent and their internal correlations and spectral structures 
(Wieland and Dalchow, 2009; Buscombe et al., 2014a). This paper addresses this shortfall by 1) detailing the implementation of computationally efficient statistical analyses of spatially distributed data such as point clouds and imagery, in the spatial and frequency domains, in such a way that the resulting statistics are themselves spatially referenced in a quasi-continuous sense (i.e. spatially explicit) as random fields of those statistical quantities; and 2) describing a new open-source toolbox for generic point cloud analysis which builds primarily on computational toolboxes for signal inference (Selig et al., 2013) and terrestrial surface analysis (Rychkov et al., 2012).

\section{The PySESA program for spatially explicit analysis of point clouds}

\subsection{Scope and purpose}

PySESA stands for 'Python program for Spatially Explicit Spectral Analysis'. Its field of application is kept broad for a burgeoning interdisciplinary community and is therefore not bound to a specific methodology or discipline. While PySESA might have immediate application in the analysis of high-resolution topographic and bathymetric point clouds, it also applies to a broad range of non-topographic, non-bathymetric, spatially-referenced data. The use of acoustic backscatter, for example, is on the ascendancy for substrate classification and bioacoustic detection (e.g. Anderson et al., 2008; Buscombe et al., 2014b; Colbo et al., 2014). Similarly, optical backscatter such as LiDAR intensities (i.e. reflectance of the LiDAR signal) is being used to facilitate terrestrial roughness and land cover classifications (e.g. Pelgrum et al., 2000; Antonarakis et al., 2008; Franceschi et al., 2009; Mallet and Bretar, 2009; Brodu and Lague, 2012; Trevisani et al., 2012), and widespread use in volcanology (e.g. Mazzarini et al., 2007), and glaciology (e.g. Arnold et al., 2006). Similar uses have been found for synthetic aperture radar (e.g. Crawford et al., 1999), colours/intensities in the visible spectrum (e.g. Carbonneau et al., 2006; Legleiter and Overstreet, 2012), or in fact any spatially referenced signal intensity in 3D or $4 \mathrm{D}$.

The input to the program is a (structured or unstructured) 'point cloud' of spatially referenced amplitudes (elevation, depth, intensity, magnitude, etc) representing any twodimensional continuous function $Z=f(X, Y)$ where $X$ and $Y$ are horizontal coordinates in a Cartesian mapping plane. Use of the term amplitude implies any relevant geophysical quantity with a spatial reference in 2 or 3 dimensions. Point cloud data are simultaneously analysed and decimated onto a regular grid. The output of the program is a set of structured, decimated point clouds of a variety of output parameters. To do so, the data are sub-divided into small windows of data with a specified degree of overlap and according to a desired output grid spacing. Each window of data is analysed statistically in either the spatial domain or frequency domain, or both. In a given window, all computed quantities are spatially co-referenced at the centroid of the $(X, Y)$ position of that window.

\subsection{Implementation}

PySESA is a command-line program implemented in platform-independent objectoriented Python ${ }^{1}$ code, with computationally demanding procedural subroutines written

\footnotetext{
${ }^{1}$ https: //www.python.org/
} 
in Cython ${ }^{2}$ (Behnel et al., 2011) using C-style static type declarations which allows compilation of static objects for efficiency. Python has become very popular for scientific computing (Oliphant, 2007; Millman and Aivazis, 2011) because it is an open-source, cross-platform, well-designed language with a clean syntax, a comprehensive standard library, and an enormous worldwide user community with free access to third-party package repositories (such as $\mathrm{PyPI}^{3}$ ). It has the immediacy of a 'scripting' style language, but also advanced capabilities such as easy interfacing with procedural languages (e.g. C or Fortran) and other object-oriented languages (e.g. C++ and Java); parallelization; graphics acceleration and distributed/cloud computing; web development; and static compiling.

Numerical computations in PySESA are built around the efficiency of the $\mathrm{NumPy}^{4}$ array (van der Walt et al., 2011), utilizing Cython's support for fast access to NumPy arrays. Additional numerical libraries are provided by $\mathrm{SciPy}^{5}$ (Jones et al., 2001-). Like recent geological and geophysical Python toolboxes (e.g. Rushing et al., 2005; Wellmann et al., 2012; Castelão et al., 2013; Krieger and Peacock, 2014) the design of PySESA is modular which allows code readability, easy extension and adaptations in the future, and the portability of its core functionality into other geospatial and geophysical analysis tools.

Operations on discrete windows of distributed data is highly amenable to so-called 'embarrassingly' parallel (Foster, 1995) processing because different CPU threads can access and process consecutive blocks of data stored in memory, without the need for communication (and/or synchronization) between the different threads. In PySESA, parallelization of computational tasks is supported using the joblib ${ }^{8}$ library which allows easy execution of tasks concurrently on (an automatically detected number of) separate CPUs. Joblib also provides special handling for efficient processing of large Numpy arrays by memory mapping using NumPy's in-built memmap ${ }^{9}$ libraries.

\subsection{Modules and typical workflow}

Implementation and installation of PySESA is described in Appendix A and some example uses are shown in Appendix B. Currently, PySESA consists of 7 main sub-modules (read for reading data into the program; partition for windowing the data into discrete portions of the input point cloud; detrend for detrending in the spatial domain and filtering in the frequency domain; spatial for calculation of statistics in the spatial domain; spectral for calculation of statistics in the frequency domain; write for writing results to file; and plot for visualisation of outputs in a variety of ways and formats). A list of all PySESA sub-modules (to date) and their functions is provided in Table 1. A typical minimal workflow (Figure 1) and associated PySESA module is as follows:

- Read 3D point cloud data into program (PySESA: :read) and specify user-inputs.

- Partition the point cloud into discrete windows of data (PySESA: :partition) according to user-specified inputs of output resolution, and degree of overlap between windows.

\footnotetext{
${ }^{2}$ http://cython.org/

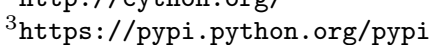

${ }^{4}$ http://www. numpy.org/

${ }^{5}$ http://www.scipy.org/

${ }^{8}$ http://pythonhosted.org//joblib/

${ }^{9}$ http://docs.scipy.org/doc/numpy/reference/generated/numpy.memmap.html
} 
- (Optional) Detrend or spatially filter each window of data (PySESA : :detrend).

- Analyse each (detrended) point cloud window for a suite of user-prescribed spatial (PySESA : spatial) and/or spectral (PySESA: :spectral) parameters.

- Output results (PySESA : : write).

- (Optional) Plot (PySESA: :plot) results in a variety of ways, using Matplotlib ${ }^{6}$ (Hunter, 2007) and Mayavi ${ }^{7}$ (Ramachandran and Varoquaux, 2011) Python modules for two- and three-dimensional graphical visualisations.

\section{Computational implementation}

\subsection{PySESA: : read}

The read module is highly optimised for reading ASCII files (comma, tab, or space delimited) composed of three columns of numbers (with floating point precision) representing $X, Y$ and $Z$, respectively. A file composed of 1 million 3D coordinates can be read into memory in less than a second with an ordinary $\approx 2.5 \mathrm{GHz}$ processor, and 10 million in less than 10 seconds.

\subsection{PySESA::partition}

Analyses are made spatially explicit by partitioning the $3 \mathrm{D}$ point cloud into small windows of data, each of which are statistically analysed and the values of user-defined parameters are assigned to the centroid location of each 3D data window. In a threedimensional region $\Omega$ consisting of points $\{P\}_{m=m_{0}}^{M}$ which is a subset of the entire point cloud $P=[X, Y, Z]$ (i.e. $\left.\{P\}_{m=m_{0}}^{M} \in P\right), \mathbb{P}_{m}$ is defined as the set in $\Omega$ consisting of those points in $P$ which are within distance $d$ of $P_{m}$ :

$$
\mathbb{P}_{m}=\mathbf{X} \in \Omega, \quad\left|\mathbf{X}-P_{m}\right|<|d|,
$$

where two-dimensional vector $\mathbf{X}=(X, Y)$ and $m=m_{0}, \ldots, M$. Given $P$ with point density $\epsilon$, the centroids of $\mathbb{P}_{m}$ are defined by (Buscombe and Rubin, 2012):

$$
P_{m}^{*}=\frac{1}{\left|\mathbb{P}_{m}\right|} \int \mathbf{X} \epsilon(\mathbf{X}) d \mathbf{X} .
$$

Here, the set of regions $\left\{\mathbb{P}_{m}\right\}_{m=m_{0}}^{M}$ are called 'windows' of $P$, and $d$ and $\{P\}_{m=1}^{M}$ can be specified in such a way that the regions overlap to a specified degree. A computationally highly efficient means to partition space as described above, with optional overlap, is a nearest-neighbour search using the $\mathrm{k}-\mathrm{d}$ (k-dimensional) tree (Bentley, 1975). In PySESA, the efficient algorithm of Maneewongvatana and Mount (1999) is implemented through SciPy's cKDTree ${ }^{10}$ function. This approach to space partitioning, as opposed to an alternative such as Voronoi tessellation (Buscombe and Rubin, 2012) or a two-pass sorting procedure (Rychkov et al., 2012), enjoys the advantages associated with easy

\footnotetext{
${ }^{6}$ http://matplotlib.org/

${ }^{7}$ http://docs.enthought.com/mayavi/mayavi/

${ }^{10}$ http://docs.scipy.org/doc/scipy/reference/generated/scipy.spatial.cKDTree.html
} 
specification of the degree of spatial smoothing (through the grid spacing and degree of overlap) in the final decimated grid. A useful feature of windowing like this is that limits can be imposed on $M$ and $m_{0}$, the maximum and minimum number of points considered, respectively, for each window.

\subsection{PySESA: : detrend}

Detrending is high-pass filtering in the spatial domain through the subtraction of a 1) mean, 2) least-squares plane, or 3) modelled surface, from the amplitude data so the small-scale variations are emphasized and the large-scale trends are removed (Brasington et al., 2012). All three approaches described above are implemented in PySESA (Figure 3). A detrending operation is a necessary pre-processing step prior to spectral analysis. Another motivation to detrend each window of data is that, as argued by Brasington et al. (2012) and Pollyea and Fairley (2011), the standard deviation of amplitudes relative to a local plane fit through the data is a more powerful statistical descriptor of amplitude roughness compared with standard deviation of $\mathbb{P}_{m}$, because it emphasises the smallest scale amplitude variance relative to the local mean amplitude (Figure 4).

Below, the detrended windowed point cloud is denoted $\widehat{\mathbb{P}_{m}}$. PySESA supports three types of plane fitting (Figure 3), those based on: 1) ordinary least squares (OLR) (e.g. Rychkov et al., 2012); 2) robust linear model (RLM); and 3) orthogonal distance regression (ODR) (e.g. Pollyea and Fairley, 2011). Given the plane through the unstructured point cloud $\mathbb{P}_{m}$, given by

$$
a X+b Y+c=0
$$

the normal vector to the plane is

$$
\mathbf{v}=\nabla f=\left[\begin{array}{l}
a \\
b \\
c
\end{array}\right] .
$$

Ordinary and robust linear regression are implemented using routines provided by the statsmodel $s^{11}$ package. In ordinary linear regression, the sum of the squared vertical distances between the $\mathbb{P}_{m}$ data values and the corresponding $\mathbb{P}_{m}$ values on the fitted plane are minimized to find $\mathbf{v}$. Robust linear models do the same via iteratively reweighted least squares and given the robust criterion estimator detailed in Huber (1981). In orthogonal distance regression (Boggs et al., 1992), $\mathbf{v}$ is found by minimizing the orthogonal (perpendicular) point-to-plane distances, $d_{i}$, given by projecting the vector from the plane to an arbitrary point $\left(x_{0}, y_{0}, \widehat{\mathbb{P}_{m}}\right)$ onto $\mathbf{v}$, a line normal to the plane

$$
d_{i}=\frac{\left|a X_{0}+b Y_{0}+\mathbb{P}_{m 0}+c\right|}{\sqrt{a^{2}+b^{2}+1}} .
$$

In PySESA, this is computed using SciPy wrappers to the ORDPACK ${ }^{12}$ library (Boggs et al., 1992) and a custom numerical procedure by which coefficients $\mathbf{v}$ from an ordinary least squares model are used as initial estimates for $\mathbf{v}$ for a more accurate fit. For very large point cloud windows, the implicit minimization of equation 3 can be speeded up considerably by pre-computing its derivatives using Jacobian functions during the fitting.

\footnotetext{
${ }^{11}$ http://statsmodels.sourceforge.net/

12 https://docs.scipy.org/doc/scipy-0.15.1/reference/odr.html
} 


\subsection{PySESA: : spectral}

3.4.1. Gridding

Gridding is the process that converts an unstructured detrended window of point cloud, $\widehat{\mathbb{P}_{m}}$, to a structured random field, $\mathbb{P}_{m}\left(\mathbf{X}_{m}\right)$, defined over the regular grid $\mathbf{X}_{m}$ composed of square grid cells, and specified by the joint probability density function $p\left(\mathbb{P}_{m}\left(\mathbf{X}_{m 1}\right), \mathbb{P}_{m}\left(\mathbf{X}_{m 2}\right), \ldots: \mathbf{X}_{m 1}, \mathbf{X}_{m 2}, \ldots \in\left[X_{m}, Y_{m}\right]\right) . \mathbb{P}_{m}\left(\mathbf{X}_{m}\right)$ consists of $N_{X_{m}} \times$ $N_{Y_{m}}$ observations at regular intervals $\Delta X_{m}=\Delta Y_{m}$ and is achieved using the SciPy routine griddata ${ }^{13}$. Nearest-neighbour interpolation (which returns the value at the data point closest to the point of interpolation) is used by default, but linear and cubic interpolation is also possible (with an associated loss in computational speed, and at the risk of introducing artificial autocorrelation into the data). Note that this process is required for spectral analyses only: descriptive statistics (section 3.8) are calculated on unstructured point clouds.

\subsubsection{Spatial Domain Filtering (with PySESA: :sgolay)}

PySESA implements the Savitsky-Golay low-pass filter (Savitzky and Golay, 1964) in $2 \mathrm{D}$ (Figure $3 \mathrm{~d}$ ) to provide the option of spatial domain filtering of $\mathbb{P}_{m}\left(\mathbf{X}_{m}\right)$ prior to spectral analysis. This can be used to low-pass filter the data or, through subtraction of the filter from the data, high-pass filter. As the latter, the Savitsky-Golay filter can also be used as a higher-dimensional detrending surface model which can be subtracted from the data instead of a 2D plane. As such, it is optionally called by the detrend module.

The idea behind Savitzky-Golay filtering is to find filter coefficients that preserve higher moments in the data. Filters such as a moving average preserve the zeroth moment of a spectrum but violate the 2nd moment. The underlying function in a Savitsky-Golay approach is approximated within a moving window by a polynomial of higher order, typically quadratic or quartic, rather than a constant. For each point $p\left(x_{m}, y_{m}\right)$ of $\mathbb{P}_{m}\left(\mathbf{X}_{m}\right)$, a window centred at that point is extracted, a least-square fit of a polynomial surface is computed, and the initial central point is replaced with the value computed by the fit. In PySESA, the coefficients are pre-computed for efficiency (using convolution routines) because they are linear with respect to the data spacing (Press et al., 2007). Evaluation of the fit at the borders of the data is achieved by padding the convolved data with a mirror image of the data.

\subsubsection{Power Spectrum}

The power spectrum $\Psi_{2}(\mathbf{K})$ (with dimensions length ${ }^{4}$ ), or equivalently its Fourier transform, the autocorrelation function $\xi_{2}(\mathbf{L})$ (over $\mathbf{L}$ lags) is a measure of the variance of amplitudes in $\mathbb{P}_{m}\left(\mathbf{X}_{m}\right)$ associated with different narrow bands of unit $\mathbf{K}=\left(k_{X}, k_{Y}\right)$, which is a two-dimensional wave vector (whose magnitude $K=\sqrt{k_{X}^{2}+k_{Y}^{2}}=2 \pi / \lambda$ is the wavenumber, $\lambda$ being the wavelength) related to the frequency components by $F_{X}=\frac{k_{X}}{N_{X} \Delta X}$ and $F_{Y}=\frac{k_{Y}}{N_{Y} \Delta Y}$. Therefore, the wavenumber describes the number of times the function $\mathbb{P}_{m}\left(\mathbf{X}_{m}\right)$ has the same phase per unit space.

To prevent spectral leakage during the estimation of $\Psi_{2}(\mathbf{K}), \mathbb{P}_{m}\left(\mathbf{X}_{m}\right)$ is first tapered by multiplying with a $2 \mathrm{D}$ taper $T(i, j)$. Then $\Psi_{2}(\mathbf{K})$ is normalized to account for the change in variance associated with the application of the taper (Buscombe et al., 2014a).

\footnotetext{
${ }^{13}$ http://docs.scipy.org/doc/scipy/reference/generated/scipy.interpolate.griddata.html
} 
Generic 2D tapering in PySESA is achieved using the vectorised method detailed in Appendix C.

Power spectral density estimation in PySESA is carried out using NIFTy ${ }^{15}$ libraries (Selig et al., 2013), capitalizing on the NIFTy rg_space ${ }^{16}$ class, which allows computationally efficient transformation between regular grid and wavenumber spaces. Power spectral density smoothing in the frequency domain is also carried out using NIFTy which implements the algorithms of Enßlin and Frommert (2011) and Oppermann et al. (2013). This process is detailed in Appendix D.

The 1D marginal spectrum, $\Psi_{1}(\mathbf{K})$, is the $2 \mathrm{D}$ spectrum collapsed as a function of the radial wavenumber $\mathbf{K}=\sqrt{K_{X}^{2}+K_{Y}^{2}}$. The subscript 1 here, and elsewhere below, denotes calculations based on the 1D form of the spectrum. No radial integration occurs, therefore this spectral form incorporates any anisotropy (directional dependence) in $\mathbb{P}_{m}\left(\mathbf{X}_{m}\right)$. If this is a concern for any reason, the user must choose a window size that ensures the spectrum is isotropic.

\subsubsection{Background Estimation}

Given the power-law form of $\Psi_{1}(\mathbf{K})$, the background spectrum, $\overline{\Psi_{1}(\mathbf{K})}$, is a version of the spectrum in which there is no concentration of variance in any wavenumber band. Comparison between $\Psi_{1}(\mathbf{K})$ and $\overline{\Psi_{1}(\mathbf{K})}$ allows identification of deviations in $\Psi_{1}(\mathbf{F})$ and therefore any statistically significant periodicities in the data. A bin-averaging approach to estimating $\overline{\Psi_{1}(\mathbf{K})}$ is biased by the peaks and troughs in the spectrum, therefore a preferable approach is to construct a simulated surface with identical global, but different local, statistics (Perron et al., 2008). In PySESA, this is achieved by simulating Gaussian $2 \mathrm{D}$ random field drawn from $\Psi_{2}(\mathbf{K})$ using the methods detailed in Oppermann et al. (2013) and summarised briefly in Appendix E, then collapsed as a function of $\mathbf{K}$ to give $\Psi_{1}(\mathbf{K})$.

This simulated field is statistically homogeneous and isotropic, which means the correlation between two field values at two positions depends only on their physical distance $\left(\left|\mathbf{X}_{m=1}-\mathbf{X}_{m=2}\right| \propto 1 / \mathbf{K}\right) . \overline{\Psi_{1}(\mathbf{K})}$ is therefore a smooth spectral approximation to an isotropic form of $\Psi_{2}(\mathbf{K})$ and has the same covariance as $\mathbb{P}_{m}\left(\mathbf{X}_{m}\right)$. This covariance captures the essential features of low-frequency variation over relatively large separation distances, but the spectra $\Psi_{1}(\mathbf{K})$ and $\overline{\Psi_{1}(\mathbf{K})}$ diverge at higher frequencies because $\overline{\Psi_{1}(\mathbf{K})}$ doesn't contain the information on either large changes in amplitude over short distances (Sayles and Thomas, 1978) or asymmetry about a vertical or horizontal axis, because is unaffected by a change in sign of $\Psi_{k=1}-\Psi_{k=2}$ or $\mathbf{X}_{m=1}-\mathbf{X}_{m=2}$ (Goff and Jordan, 1988).

\subsection{Integral lengthscale (with PySESA: : lengthscale)}

The autocorrelation function is the normalised covariance between the signal and itself when offset by some lag, and exhibits periodicity - where present - at the same period as the original signal. In PySESA, the autocovariance function $\xi_{2}(\mathbf{L})$, over $\mathbf{L}$ lags, is calculated as the $2 \mathrm{D}$ continuous Fourier transform of $\Psi_{2}(\mathbf{K})$ (Priestley, 1981) then integrated radially over segments to collapse it to $1 \mathrm{D}$ :

\footnotetext{
${ }^{15}$ http://www.mpa-garching.mpg.de/ift/nifty/index.html

${ }^{16}$ http://www.mpa-garching.mpg.de/ift/nifty/base_space.html
} 


$$
\xi_{1}(\mathbf{L})=\int_{0}^{2 \pi} \mathscr{F}\left[\Psi_{2}(\mathbf{K})\right](\mathbf{K} \cos \theta, \mathbf{K} \sin \theta) \mathbf{K} d \theta,
$$

where $\theta$ is a vector of equal-area sectors subtended by a given angle centred in the DC component in frequency space, over which the radial integration occurs. It is assumed that the radial integration incorporates any significant anisotropy in $\mathbb{P}_{m}\left(\mathbf{X}_{m}\right)$.

The definition of the integral length-scale, $l_{0}$, comes originally from turbulence research (Taylor, 1938) as a measure of some relatively large lag over which the the autocorrelation converges to zero, indicative of the largest turbulent eddy scale. The same principle applies to spatially distributed data if fluctuating velocity in time is replaced by fluctuating amplitude in space (c.f. Nikora, 2005). Strictly speaking, $l_{0}$ is the product of $2 \pi$ and the spectral amplitude at $\mathbf{K}=0$ (Taylor, 1938) however evaluation of this amplitude would require an infinitely long spatial series. A pragmatic approach is to pick the lag to which, when integrated to, the correlation equals zero (beyond which only harmonics remain, whose correlations by definition are harmonics at the same wavenumber), or:

$$
l_{0}=\int_{\mathbf{L}_{0}} \xi_{1}(\mathbf{L}) d \mathbf{L},
$$

with $\mathbf{L}_{0}$ defined as either the lag to which $\xi_{1}$ falls to zero (Taylor, 1938), the product of $2 \pi$ and the lag at which $\xi_{1}$ falls to half its value at zero lag (Buscombe et al., 2010), or the lag required to reduce $\xi_{1}$ to $1 / e$ (Shepard et al., 2001). All three methods for calculating the integral lengthscale are common and provided in PySESA and it is left as an exercise to the interested reader to examine how these measures relate for different point clouds.

In general, smoother surfaces have larger integral lengthscales. However, the concepts behind this statistical measure have been used to describe how variance in various geophysical phenomena cascades (dissipates, or 'smears' (Jerolmack and Paola, 2010)) across spatial scales (Guadagnini and Neuman, 2011), in which case large integral lengthscales could also indicate slow 'dissipation' rates from variance associated with small wavelengths to variances associated with larger wavelengths in the data.

\subsubsection{Slope and intercept}

Spectral power of distributed spatial data decreases rapidly with increasing frequency (Shepard et al., 2001). This power-law behaviour cannot persist at very high frequencies, which leads to spectral 'roll over' where the spectral slope steepens (Priestley, 1981). The length scale associated with this rollover frequency is the outer scale $L_{0}$, which is assumed in PySESA to be the point of divergence between $\Psi_{1}\left(\mathbf{X}_{m}\right)$ and the background power spectrum $\overline{\Psi_{1}(\mathbf{K})}$. A simple functional form of $\Psi_{1}(\mathbf{K})$ is a power-law (von Karman and Howarth, 1938):

$$
\widehat{\Psi_{1}(\mathbf{K})}=\frac{\omega_{1}}{\left(h_{0}|\mathbf{K}|\right)^{\gamma_{1}}}, \quad \frac{2 \pi^{-1}}{\mathbf{K}}>1 / L_{0},
$$

The inclusion of a dimensional constant, $h_{0}$, in equation 8 allows $\omega_{1}$ to have dimensions dimensions length ${ }^{4}$, independent of the value of non-dimensional $\gamma_{1}$ (Jackson and 
Richardson, 2007). Spectral strength and exponent are estimated from bin averages of the marginal power spectrum $\Psi_{1}(\mathbf{K})$, as the parameters that minimize the error

$$
\left\|\left(\gamma_{1} \mathbf{K}_{b}+\omega_{1}\right)+\widehat{\Psi_{1 b}}\right\|^{2}, \quad \mathbf{K}_{b}=\frac{2 \pi^{-1}}{\mathbf{K}}>1 / L_{0},
$$

where || represents the 2-norm and subscript $b$ denotes bin average. Appendix F details the parameter estimation. As well as $\gamma_{1}$ and $\omega_{1}$, the correlation coefficient of the regression, the two-sided p-value for a hypothesis test whose null hypothesis is that the slope is zero, and the standard error of the slope coefficient estimate $\left(=\sqrt{M S E / \sigma_{\mathbf{K}_{b}}}\right.$ where $M S E$ is a mean square error - the sum of squared residuals divided by number of model parameters - and $\sigma_{\mathbf{K}_{b}}$ is the variance in the independent variable) are also calculated. Since $\gamma_{1}$ is always negative, an estimate of fractal dimension is then $D=\left(8+\gamma_{1}\right) / 2$ (Huang and Turcotte, 1990; Perron et al., 2008).

The spectral strength, $\omega_{1}$, is a measure of power at low frequencies, or the magnitude of signal fluctuations over relatively large spatial distances. The spectral exponent, $\gamma_{1}$, is a measure of the rate of decay in signal power as a function of increasing frequency. The more complex the spatial patterns in the data, the greater range of frequencies must be used to describe it. Therefore, $\gamma_{1}$ is a useful measure of how complex the data is by quantifying the range of frequencies necessary to describe the data.

\subsection{Amplitude and length scales}

The area under the power spectral density curve is equal to the variance of the amplitude distribution (Sayles and Thomas, 1978). For normally distributed amplitudes, $\sigma_{1}$ is equivalent to the root-mean-square amplitude, which in PySESA is calculated as:

$$
\sigma_{1}=\sqrt{\int_{\mathbf{K}_{0}} \Psi_{1}(\mathbf{K}) d \mathbf{K}}, \quad \frac{2 \pi^{-1}}{\mathbf{K}_{0}}=\frac{2 \pi^{-1}}{\mathbf{K}}>1 / L_{0},
$$

in which the definite integral is estimated using the composite trapezoidal method (SciPy's trapz function). $\sigma_{1}$ is a measure of the magnitude of signal fluctuations over all space (both large and small separation distances) and is therefore only pertinent to roughness, not texture, which is better quantified by measures of dominant wavelengths in the data. PySESA calculates peak wavelength as:

$$
\lambda_{\text {peak }}=\left(\frac{2 \pi}{\mathbf{K}\left[\operatorname{argmax}\left(\Psi_{1}(\mathbf{K}) / \overline{\Psi_{1}(\mathbf{K})}\right)\right]}\right) d \mathbf{X},
$$

which can only take on discrete values. A more continuously distributed measure of central tendency in wavelength is also calculated:

$$
\lambda_{\text {mean }}=\int_{K_{0}}\left(\frac{\Psi_{1}(\mathbf{K})}{\overline{\Psi_{1}(\mathbf{K})}}\right) 2 \pi^{-1} d \mathbf{X}, \quad \frac{2 \pi^{-1}}{\mathbf{K}_{0}}=\frac{2 \pi^{-1}}{\mathbf{K}}>1 / L_{0} .
$$

The ratio of the RMS roughness (equation 10) to the integral lengthscale gives the 'effective slope' (Campbell and Garvin, 1993; Shepard et al., 2001), expressed in degrees:

$$
\phi=\tan ^{-1}\left(\frac{\sigma_{1}}{l_{0}}\right) .
$$




\subsection{Moments and Spectral Width}

PySESA provides the means to calculate a number of useful quantities from the moments of the power spectrum $\Psi_{1}(\mathbf{K})$, defined as:

$$
m_{k}=\int_{0}^{\infty} \mathbf{K}^{k}\left|\Psi_{1}(\mathbf{K})\right|^{2} d \mathbf{K},
$$

which says that the content at every frequency in the spectrum is weighted by the $k$ th power of the frequency and the result is summed up across the entire spectrum. The power in the signal is $m_{0}$. The moment of inertia around the axis $\mathbf{K}=0$ is $m_{2}$. Since the bandwidth of the signal is $\sigma_{m}=\sqrt{m_{2} / m_{0}}$, the number of zero crossings per unit space is given by $N_{0}=2 \sqrt{m_{2} / m_{0}}$. The derivative of $\mathbb{P}_{m}\left(\mathbf{X}_{m}\right)$ has the marginal power spectrum $\left|2 \pi \mathbf{K} \Psi_{1}(\mathbf{K})\right|^{2}$ and the bandwidth $\sqrt{m_{4} / m_{2}}$, therefore the number of extrema per unit space is $E_{0}=2 \sqrt{m_{4} / m_{2}}$. Two measures of the average wavenumber are $\lambda=m_{0} / m_{1}$ and $\lambda=\sqrt{m_{0} / m_{2}}$. The spectral width is a dimensionless parameter which describes the way in which spectral area is distributed around the mean wavenumber. Two measures of spectral width are implemented in PySESA: 1) $\nu=\sqrt{1-m_{2}^{2} / m_{0} m_{4}}$ (Cartwright and Longuet-Higgins, 1956) which approaches zero as the spectrum becomes more narrow banded; and 2) the 'normalised radius of gyration', or $\nu=\sqrt{\left(m_{0} m_{2} / m_{1}^{2}\right)-1}$ (LonguetHiggins, 1975) which doesn't rely on the fourth spectral moment, so is numerically more stable.

\subsection{PySESA::spatial}

PySESA is predominantly a library for spectral analyses but also implements operations for calculation of descriptive statistics (standard deviation, skewness, and kurtosis) on point clouds in the spatial domain. Root-mean-square (RMS) height, or the standard deviation of amplitudes about the mean, is the square root of the variance of amplitudes

$$
\sigma^{2}=\left\langle\left(\mathbb{P}_{m}(Z)-\overline{\mathbb{P}_{m}(Z)}\right)^{2}\right\rangle
$$

or detrended amplitudes

$$
\sigma_{d}^{2}=\left\langle\left(\widehat{\mathbb{P}_{m}}(Z)-\widehat{\mathbb{P}_{m}}(Z)\right)^{2}\right\rangle
$$

Sample variance, skewness and kurtosis are calculated using the numerically stable method of Welford (1962) as implemented by Knuth (1998) and discussed by Chan et al. (1983). This method is less prone to loss of precision in floating point arithmetic due to subtracting two nearly equal numbers, which is especially important when calculating the variance of small residuals of points relative to a plane. Large errors in compiled statistics can result otherwise. The Welford-Knuth algorithm is written in C++ and compiled into a Python module using the SWIG ${ }^{17}$ interface compiler (Beazley, 2003). The 'effective slope' (ratio of the RMS roughness to the integral lengthscale) can be calculated in the spatial domain using equation 13.

\footnotetext{
${ }^{17}$ www.swig.org/exec.html
} 


\section{Demonstration}

In order to demonstrate the functionality of the PySESA toolbox, a bathymetric point cloud of a $60 \times 80 \mathrm{~m}$ patch of the Colorado River bed in Western Grand Canyon (Figure 5), around river mile 224 (approximately $360 \mathrm{~km}$ downstream of Lees Ferry, Arizona, USA) was analysed. The point cloud was obtained using multibeam echosounder, is composed of almost 1 million 3D points (at a density of around 200 points per square metre). Details on the methods for acquisition and analysis of such data in this environment are found in Kaplinski et al. (2009, 2014), Grams et al. (2013) and Buscombe et al. (2014a). Most important for the present purposes is that the point cloud clearly shows areas of varying textures and roughnesses, including sand dunes with a quasi-regular crest spacing, relatively flat sand areas, and relatively high elevation rocky areas. The point cloud was analysed for all spatial and spectral parameters using a $0.25 \times 0.25 \mathrm{~m}$ regular output grid spacing with $0 \%$ overlap. Each window contained a minimum of 64 data points. A ODR plane was used to detrend data in each window. Prior to spectral analysis, the data were Hann tapered.

The decimated output point cloud shown in Figure 6a has been colour-coded by spectral root-mean-square variation in amplitude, $\sigma_{1}(\mathrm{~m})$ (equation 10). As expected, roughness is high (light colours) in the rocky areas, intermediate in the dune field, and low in the flatter areas in between. The same cloud of points in Figure $6 \mathrm{~b}$ has been colour-coded by spectral strength $\omega_{2}\left(\mathrm{~m}^{4}\right)$ (equation 9 ). To recap from section 3.5.1, the spectral strength, $\omega_{1}$, is a measure of power at low frequencies. Rocky areas therefore have relatively low values of spectral strength because the magnitude of topographic fluctuations over relatively large spatial distances is small compared to those over short distances. The potential for automated physically-based segmentation of different geomorphic units (dunes, flat sand and rocks) is apparent in this case and would have enormous potential application in, for example, channel bed physical habitat characterisation and sediment transport studies. To further illustrate this point, contour maps of various gridded parameters, which are a selection of those resulting from spatial and spectral analyses of the point cloud shown in Figure 5 are shown in Figure 7 . In each subplot, just a small $70 \times 45 \mathrm{~m}$ portion of the data is shown. Spectral strength (Figure 7b), spectral width (Figure 7e), ODR detrended standard deviation (Figure 7f) and ratio of integral lengthscale and RMS roughness (Figure 7i) would be particularly effective parameters by which to delineated rocky, flat and rough sand areas. Other parameters such as the non-detrended standard deviation (Figure $7 \mathrm{f}$ ) and integral lengthscale (Figure 7d) seem likely to be able to delineate dune crests from troughs.

Similar analyses could find particular utility in, for example, automated landscape, soil or vegetation classification or segmentation of natural textures in remote sensing imagery; seafloor substrate mapping and benthic habitat characterisation using multibeam data; or spatially explicit mapping of grain size and roughness variations in streambeds, surficial geology, lava flows or vertical sedimentary sequences using LiDaR or highresolution imagery, among many other uses.

\section{Discussion and future developments}

According to Trevisani et al. (2012) and Berti et al. (2013), an ideal algorithm for a spatially explicit analysis of surfaces should: 
(1) provide a pixel-by-pixel characterisation of the surface;

(2) run on large datasets with a computational and memory efficiency;

(3) measure an intrinsic property of the surface, invariant with respect rotation or translation;

(4) take into account scale dependency; and

(5) have an intuitive or physical meaning.

It is instructive to evaluate the PySESA toolbox against these criteria. (1) doesn't strictly apply because geospatial data are analysed as point clouds rather than gridded surfaces, however information from each measured location in the point cloud is utilized. There is no interpolation across space: if there is no data in a particular grid location, or not enough data (defined by the min_pts parameter to the partition module), there are no outputs at that location. The spatial density of results (degree of decimation) depends on the (user-defined) scale at which the outputs are meaningful, and the processing time (related to the size of the cloud) deemed acceptable.

Regarding (2), special attention has been paid to making the program computationally efficient (within the constraints of using an interpreted language) using statically compiled subroutines which run in parallel. So far, the program has been used on up to and including $\mathcal{O}\left(10^{7}\right)$ point clouds. More work is required to make the program memory efficient enough to process point clouds of $\mathcal{O}\left(10^{8}\right)$ or more. The program would run with only minor modifications on high performance computing environments. The combination of a one-time binary-tree (k-d tree) space partition, with a computational complexity $\mathcal{O}(n \log n)$, to sort the point cloud into windows, then successive application of the FFT algorithm, each with a computational complexity $\mathcal{O}(n \log n)$, on each window, results in an overall computational cost of $\mathcal{O}\left(n^{2}\right)$ to analyse each point cloud. Therefore the overall processing time as a function of the number of points in the cloud is quasi-linear in log-log space (Figure 8) and doubling the number of processors over which the computations are handled results in a $\approx 50 \%$ speedup (Figure 9 ).

Metrics calculated using Fourier methods are not inherently invariant with respect to rotation or translation (3). However, because small windows are used in the processing; because detrending can be applied; and because spectral metrics computed in PySESA are based on 2D spectra which are then collapsed (not radially averaged) to a 1D form; any anisotropy is incorporated. The one caveat to that statement would be for coarse output grids. How coarse is too coarse depends on the degree of anistropy in a typical data window. In choosing an appropriate window size (a function of output spacing and overlap), there is a trade-off between a size small enough to ensure data in a typical window are isotropic, yet large enough to preserve required detail in the outputs at an acceptable statistical power (related to $N$ ). The effects of window size and degree of window overlap would vary on the degree of spatial variability in the data, and on the specific output parameter. Those parameters quantifying lengthscales (e.g. $\lambda_{\text {mean }}$ and $l_{0}$ ) are most susceptible to choice of window size, but large window sizes also affect measures of amplitude (e.g. $\sigma_{1}, \sigma$ and $\sigma_{d}$ ) if amplitudes are strongly varying across the window such that mean or plane detrending has a diminished effect of amplifying local variations in amplitude relative to the mean amplitude. Window overlap controls the degree of spatial smoothing in the outputs and therefore its effects on output parameters is hard to predict. 
On (4), as discussed in section 1.3, spectral methods provide the means to calculate horizontal (e.g. $\lambda_{\text {mean }}$ and $l_{0}$ ) and amplitude (e.g. $\left.\sigma_{1}\right)$ scaling and the scaling between them (such as $D$ and $\phi$ ). Finally, regarding (5), all measures calculated by the PySESA (summarised in Table 1) have physical connotations (indeed, most have physical units), being related to either the amplitude or horizontal lengthscales of signal fluctuations or measures describing the distribution of amplitudes in the spatial (e.g. skewness and kurtosis) or frequency (e.g. $m_{k}$ and $\nu$ ) domains.

PySESA could be extended by inclusion of frequency domain filtering and bandwidth specification which would allow the user to specify a range of wavenumbers over which to calculate the power spectrum. In addition, co-variance and co-spectra of $4 \mathrm{D}$ data (two dependent amplitudes variables co-registered in space) such as lidar intensities and elevations, or sonar backscatter amplitudes and depths, could be calculated. Finally, the toolbox could be easily extended to include spatial analogs to the power spectrum such as variograms and structure functions.

\section{Acknowledgements}

This work was funded by the Glen Canyon Dam Adaptive Management Program administered by the U.S. Bureau of Reclamation. Any use of trade, product, or firm names is for descriptive purposes only and does not imply endorsement by the U.S. government. Thanks to Matt Kaplinski, Erich Mueller and Bob Tusso for helping collect the field data, and Paul Grams for helpful discussions.

\section{Appendix A. Implementation and installation}

- PySESA is completely open source and has been developed under a GNU General Public License. The project homepage is http://dbuscombe-usgs.github.io/ pysesa/ which provides documentation and further analysis examples.

- The program requires NumPy, SciPy, Cython, matplotlib, NIFTy, joblib, and statsmodels modules. A setup.py distutils ${ }^{18}$ script is provided to automatically install these dependencies.

- The program is available on the Python package repository (https://pypi . python. org/pypi/pysesa) and can be installed from the command line using: pip install pysesa.

- The ASCII format is used for both input and outputs, despite the overhead involved in textural conversions and the sequential nature of I/O operations, for maximum compatibility with other software. Support for other, more efficient, binary formats (such as LAS, netCDF and HDF) will be implemented in the future to the read module.

\footnotetext{
${ }^{18}$ https://docs.python.org/2/distutils/
} 
- PySESA has a git version-control backend and is freely available on the github ${ }^{\circledR}$ online repository: https://github.com/dbuscombe-usgs/pysesa which allows centralised storage and customization by users ('forking') through development branches ('forks'). Additions of new functions and sub-modules can be made or incorporated into other software tools by interested developers.

- Each function is annotated with docstrings explaining functionality and syntax, which can be accessed within python using module._-doc_-_, or using the module? syntax in ipython ${ }^{19}$.

- $\operatorname{sphin} x^{20}$ has been used to generate html web pages for the project. These can be compiled locally using the supplied Makefile (make html) or batch (make.bat) file on Windows ${ }^{\circledR}$.

- So far the program has been tested with Python version 2.7, on various distributions of Linux and Windows ${ }^{\circledR} 7$.

\section{Appendix B. Example usages of PySESA}

The submodule PySESA : : process allows full control over all types of workflows through use of a number of processing flags. A minimum working example usage of the the PySESA module, accepting all default values for parameters, is:

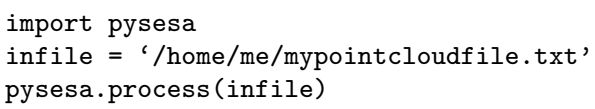

This instance writes out the following results file whose name contains some of the processing parameters:

/home/me/mypointcloudfile.txt_zstat_detrend4_outres0.5_proctype1_mxpts512_minpts16.xyz

The above is the same as passing a list of default-valued variables to PySESA : : process, which is included for completeness in the PySESA : : test module:

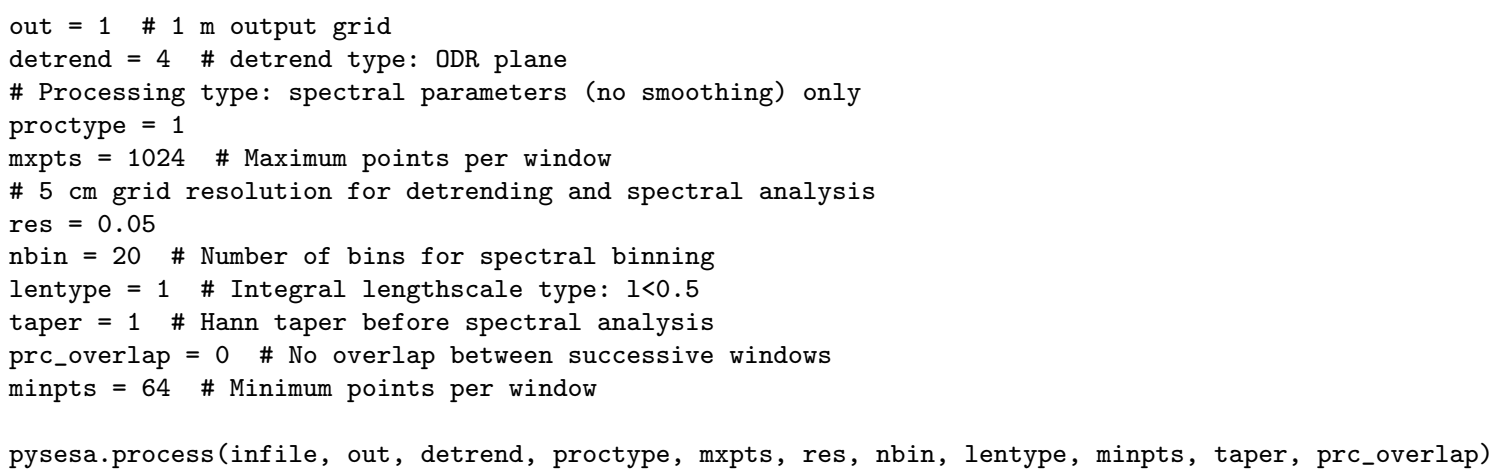


A minimal example analysis of spatial and spectral analysis on just 1 window of data:

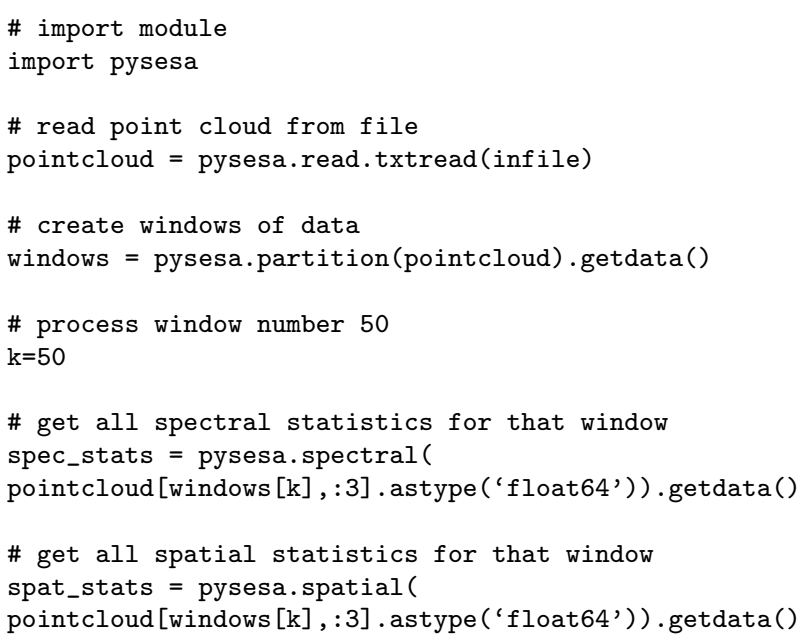

and to extend this to all windows, utilising parallel processing over all available cores, could be achieved using the following minimal example:

\# define a function that will get repeatedly

\# read by the parallel processing queue

def get_spat_n_spec(pts):

return pysesa.spatial (pts.astype('float64')).getdata()

+ pysesa.spectral (pts.astype('float64')).getdata()

\# import the parallel processing libraries

from joblib import Parallel, delayed, cpu_count

\# Processing type: spatial plus spectral

\#parameters (no smoothing)

proctype $=4$

\# process each window with all available cores,

\# by queueing each window in a sequence

\# and processing until they are all done

$\mathrm{w}=$ Parallel ( $\mathrm{n}_{-} \mathrm{jobs}=\mathrm{cpu}$ _count ()$\left., \operatorname{verbose}=0\right)$

(delayed (get_spat_n_spec) (pointcloud [windows [k], :3])

for $\mathrm{k}$ in $\mathrm{xrange}(\operatorname{len}($ windows))

\# parse out the outputs into variables

$\mathrm{x}, \mathrm{y}, \mathrm{z}_{-}$mean, z_max, z_min, z_range, sigma, skewness, ...

kurtosis, $n$, slope, intercept, r_value, p_value, ...

std_err, d, 1, wmax, wmean, rms1, rms2, Z, E, ...

sigma, T0_1, T0_2, sw1, sw2, m0, m1, m2, ..

$\mathrm{m} 3, \mathrm{~m} 4, \operatorname{phi}=\operatorname{zip}\left(*_{\mathrm{w}}\right)$

To obtain just the integral lengthscale of the $\mathrm{kth}$ window, detrended using the orthogonal distance regression detrending technique, one could use:

detrend $=4$ \# Orthogonal distance regression

pysesa.lengthscale (pysesa.detrend (

pointcloud [windows [k] , :3], detrend).getdata()).getlengthscale() 
629

630

and to get the spatial statistics from the same data:

pysesa.spatial (pysesa.detrend (

pointcloud [windows [k] , :3], detrend) $\cdot \operatorname{getdata(})) \cdot \operatorname{getdata}()$

In this final example, the output grid resolution is changed to $25 \mathrm{~cm}$, and the various outputs from the spectral module are obtained separately:

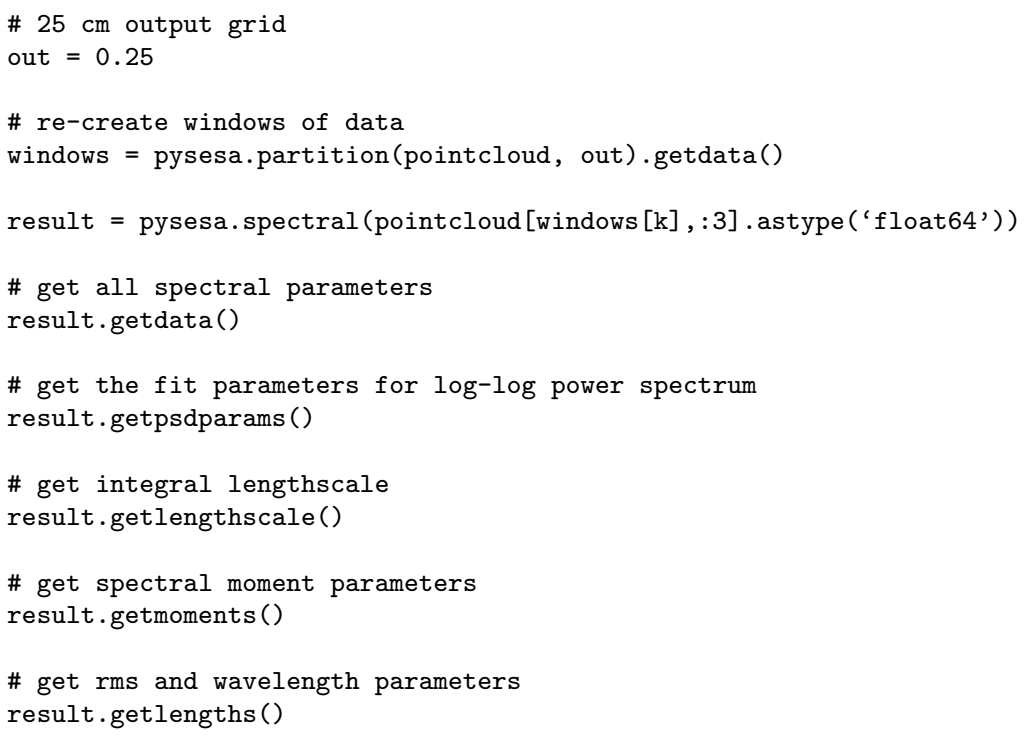

\section{Appendix C. Two dimensional tapering}

A vectorised implementation of a $2 \mathrm{D}$ taper is the outer product of two $1 \mathrm{D}$ vectors (below denoted $A$ and $B$ ) describing window functions of lengths $i$ and $j$, respectively:

$$
T(i, j)=\sqrt{\left[\begin{array}{cccc}
A_{0} \cdot B_{0} & A_{0} \cdot B_{1} & \ldots & A_{0} \cdot B_{j} \\
A_{1} \cdot B_{0} & A_{1} \cdot B_{1} & \ldots & A_{1} \cdot B_{j} \\
\vdots & \vdots & \vdots & \vdots \\
A_{i} \cdot B_{0} & A_{i} \cdot B_{1} & \ldots & A_{i} \cdot B_{j}
\end{array}\right] .}
$$

This approach is is both highly optimised and allows implementation of any custom (user-defined) 1D window function for tapering. Currently, the NumPy taper functions hanning (raised cosine), hamming (weighted cosine), bartlett (triangular) and blackman are implemented.

\section{Appendix D. Spectral smoothing.}

The smoothing of power spectral density, $\Psi(\mathbf{K})$ is bin averaged, padded, then convolved with the Gaussian kernel $g=e^{-2 \pi^{2} \mathbf{K}^{2} d \mathbf{K}^{2}}$ through application of the convolution theorem, such that 


$$
\mathscr{F}\left\{\Psi_{2}(\mathbf{K}) \times g\right\}=\mathscr{F}\left\{\Psi_{2}(\mathbf{K})\right\} \cdot \mathscr{F}\{g\},
$$

where $\mathscr{F}$ denotes Fourier transform. Then the inverse Fourier transform is applied, the padding removed, and the absolute value taken as the smoothed power spectrum. This approach takes computational advantage of the fact that smoothing power spectrum with the kernel then taking derivatives is equivalent to smoothing power spectrum directly with the derivative of the kernel (Lashermes et al., 2007), or

$$
\frac{\delta}{\delta \mathbf{K}}\left(\Psi_{2}(\mathbf{K}) \times g\right)=\Psi_{2}(\mathbf{K}) \times \frac{\delta g}{\delta \mathbf{K}} .
$$

\section{Appendix E. Background power spectrum.}

To summarise briefly, Gaussian random fields are fields drawn from a multivariate normal distribution that is characterized by its mean and covariance. A Hermitian random field is drawn from a Gaussian distribution with power spectrum $\Psi(\mathbf{K})$ :

$$
H\left(\mathbf{X}_{m}\right)=\frac{\mathscr{F}\left(G\left(\mathbf{X}_{m}\right)\right)}{\sqrt{X_{m} Y_{m}}} \sqrt{\Psi_{2}(\mathbf{K})},
$$

where $G\left(\mathbf{X}_{m}\right)$ is a matrix of realisations drawn from a Gaussian $(\mu=0, \sigma=1)$ probability distribution function. The random field is the given as the real part of inverse Fourier transform of $H\left(\mathbf{X}_{m}\right)$, shifted so the the zero-frequency component is at the centre of the spectrum. The background spectrum is calculated in 2D from the Gaussian field.

\section{Appendix F. Spectral slope and intercept.}

The parameter vector $\left(\gamma_{1}, \omega_{1}\right)^{t}$, where $t$ indicates transpose, is calculated as the leastsquares solution of the following over-determined linear system

$$
\left[\begin{array}{rr}
\log 10\left[\mathbf{K}_{1}\right] & 0 \\
\log 10\left[\mathbf{K}_{2}\right] & 0 \\
\vdots & \\
\log 10\left[\mathbf{K}_{b}\right] & 0
\end{array}\right]\left(\gamma_{1}, \omega_{1}\right)^{t}=\left[\begin{array}{r}
\log 10\left[\widehat{\Psi_{11}}\right] \\
\log 10\left[\widehat{\Psi_{12}}\right] \\
\vdots \\
\log 10\left[\widehat{\Psi_{1 b}}\right]
\end{array}\right]
$$

which is solved using the robust linear regression routine provided by the statsmodels module.

\section{References}

Aberle, J., Nikora, V., Henning, M., Ettmer, B., Hentschel, B., 2010. Statistical characterization of bed roughness due to bed forms: A field study in the Elbe River at Aken, Germany. Water Resources Research 46, W03521.

Anderson, J.T., Holliday, D.V., Kloser, R., Reid, D.G., Simard, Y., 2008. Acoustic seabed classification: current practice and future directions. ICES Journal of Marine Science 65, 1004-1011.

Antonarakis, A.S., Richards, K.S., Brasington, J., 2008. Object-based land cover classification using airborne LiDAR. Remote Sensing of the Environment 112, 2988-2998.

Antonarakis, A.S., Richards, K.S., Brasington, J., Bithell, M., 2009. Leafless roughness of complex tree morphology using terrestrial LiDAR. Water Resources Research 45, W10401. 
Arnold, N.S., Rees, W.G., Devereux, B.J., Amable, G.S., 2006. Evaluating the potential of high resolution airborne LiDAR data in glaciology. International Journal of Remote Sensing 27, 1233-1251. doi:10. 1080/01431160500353817.

Balmino, G., 1993. The spectra of the topography of the Earth, Venus and Mars. Geophysical Research Letters 20, 1063-1066.

Beazley, D.M., 2003. SWIG: An extensible compiler for creating scriptable scientific software, in: Future Generation Computer Systems (FGCS) 19. Elsevier, Amsterdam, pp. 599-609.

Behnel, S., Bradshaw, R., Citro, C., Dalcin, L., Seljebotn, D.S., Smith, K., 2011. Cython: The best of both worlds. Computing in Science and Engineering 13, 31-39. doi:10.1109/MCSE.2010.118.

Bentley, J.L., 1975. Multidimensional binary search trees used for associative searching. Communications of the ACM 18, 509-517.

Berti, M., Corsini, A., Daehne, A., 2013. Comparative analysis of surface roughness algorithms for the identification of active landslides. Geomorphology 182, $1-18$. doi:http://dx.doi.org/10.1016/j . geomorph.2012.10.022.

Boggs, P.T., Byrd, R.H., Rogers, J.E., Schnabel, R.B., 1992. User's reference guide for ORDPACK version 2.01 software for weighted orthogonal regression. Technical Report. U.S. Department of Commerce Applied and Computational Mathematics Division. Gaithersburg. URL: http: //docs.scipy.org/doc/external/odrpack_guide.pdf.

Booth, A.M., Roering, J.J., Perron, J.T., 2009. Automated landslide mapping using spectral analysis and high-resolution topographic data: Puget Sound lowlands, Washington, and Portland Hills, Oregon. Geomorphology 109, 132-147.

Brasington, J., Vericat, D., Rychkov, I., 2012. Modeling river bed morphology, roughness, and surface sedimentology using high resolution terrestrial laser scanning. Water Resources Research 48, W11519.

Brodu, N., Lague, D., 2012. 3D terrestrial LiDAR data classification of complex natural scenes using a multi-scale dimensionality criterion: Applications in geomorphology. ISPRS Journal of Photogrammetry and Remote Sensing 68, 121-134.

Buckley, S.J., Howell, J.A., Enge, H.D., Kurz, T.H., 2008. Terrestrial laser scanning in geology: data acquisition, processing and accuracy considerations. Journal of the Geological Society of London 165 625-638. doi:10.1144/0016-76492007-100.

Burrough, P., van Gaans, P., MacMillan, R., 2000. High-resolution landform classification using fuzzy k-means. Fuzzy Sets and Systems 113, 37-52

Buscombe, D., 2013. Transferable wavelet method for grain size-distribution from images of sediment surfaces and thin sections, and other natural granular patterns. Sedimentology 60, 1709-1732.

Buscombe, D., Grams, P.E., Kaplinski, M.A., 2014a. Characterizing riverbed sediments using highfrequency acoustics 1: Spectral properties of scattering. Journal of Geophysical Research - Earth Surface 119, doi:10.1002/2014JF003189

Buscombe, D., Grams, P.E., Kaplinski, M.A., 2014b. Characterizing riverbed sediments using highfrequency acoustics 2: Scattering signatures of Colorado River bed sediments in Marble and Grand Canyons. Journal of Geophysical Research - Earth Surface 119, doi:10.1002/2014JF003191.

Buscombe, D., Rubin, D.M., 2012. Advances in the simulation and automated measurement of wellsorted granular material: 1. Simulation. Journal of Geophysical Research - Earth Surface 117, F02001.

Buscombe, D., Rubin, D.M., Warrick, J.A., 2010. A universal approximation to grain size from images of non-cohesive sediment. Journal of Geophysical Research - Earth Surface 115, F02015.

Campbell, B.A., Garvin, J.B., 1993. Lava flow topographic measurements for radar data interpretation. Geophysical Research Letters 20, 831-834. doi:10.1029/93GL00737.

Carbonneau, P.E., Bergeron, N., Lane, S.N., 2005. Automated grain size measurements from airborne remote sensing for long profile measurements of fluvial grain sizes. Water Resources Research 41, W11426. doi:10.1029/2005WR003994.

Carbonneau, P.E., Lane, S.N., Bergeron, N.E., 2006. Feature based image processing methods applied to bathymetric measurements from airborne remote sensing in fluvial environments. Earth Surface Processes and Landforms 31, 1413-1423.

Cartwright, D.E., Longuet-Higgins, M.S., 1956. The statistical distribution of the maxima of a random function. Proceedings of the Royal Society of London Series A. 237, 212-232.

Castelão, G.P., L. C. Irber, J., Boas, A.V., 2013. An objective reference system for studying rings in the ocean. Computers and Geosciences 61, 43-49.

Cataño-Lopera, Y.A., Abad, J.D., García, M.H., 2009. Characterization of bedform morphology generated under combined flows and currents using wavelet analysis. Ocean Engineering 36, 617-632.

Chan, T.F., Golub, G.H., LeVeque, R.J., 1983. Algorithms for computing the sample variance: Analysis and recommendations. The American Statistician 37, 242-247. 
Church, E.L., 1988. Fractal surface finish. Applied Optics 27, 1518-1526.

Colbo, K., Ross, T., Brown, C., Weber, T., 2014. A review of oceanographic applications of water column data from multibeam echosounders. Estuarine, Coastal and Shelf Science 145, $41-56$.

Crawford, M., Kumar, S., Ricard, M., Gibeaut, J., Neuenschwander, A., 1999. Fusion of airborne polarimetric and interferometric SAR for classification of coastal environments. IEEE Transactions on Geoscience and Remote Sensing 37, 1306-1315.

Dassot, M., Constant, T., Fournier, M., 2011. The use of terrestrial LiDAR technology in forest science: application fields, benefits and challenges. Annals of Forest Science 68, 959-974.

Enßlin, T., Frommert, M., 2011. Reconstruction of signals with unknown spectra in information field theory with parameter uncertainty. Physical Review D 83, 105014. doi:10.1103/PhysRevD.83.105014.

Family, F., 1986. Scaling of rough surfaces: effects of surface diffusion. Journal of Physics A: Mathematical and general 19, L441.

Fara, H.D., Scheidegger, A.E., 1961. Statistical geometry of porous media. Journal of Geophysical Research 66, 3279-3284.

Fonstad, M.A., Dietrich, J.T., Courville, B.C., Jensen, J.L., Carbonneau, P.E., 2013. Topographic structure from motion: a new development in photogrammetric measurement. Earth Surface Processes and Landforms 38, 421-430.

Foster, I., 1995. Designing and building parallel programs. doi:ISBN:9780201575941. section 1.4.4. AddisonWesley.

Fox, C.G., Hayes, D.E., 1985. Quantitative methods for analyzing the roughness of the seafloor. Reviews of Geophysics 23, 1-48.

Franceschi, M., Teza, G., Preto, N., Pesci, A., Galgaro, A., Girardi, S., 2009. Discrimination between marls and limestones using intensity data from terrestrial laser scanner. ISPRS Journal of Photogrammetry and Remote Sensing 64, 522-528.

Frankel, K.L., Dolan, J.F., 2007. Characterizing arid region alluvial fan surface roughness with airborne laser swath mapping digital topographic data. Journal of Geophysical Research - Earth Surface 112, F02025.

Furbish, D.J., 1987. Conditions for geometric similarity of coarse stream-bed roughness. Mathematical Geology 19, 291-307.

Gilman, D.L., Fuglister, F.J., J. M. Mitchell, J., 1963. On the power specrum of 'red noise'. Journal of Atmospheric Science 20, 182-184.

Glenn, N.F., Streutker, D.R., Chadwick, D.J., Thackray, G.D., Dorsch, S.J., 2006. Analysis of LiDARderived topographic information for characterizing and differentiating landslide morphology and activity. Geomorphology 73, 131-148.

Goff, J.A., Jordan, T.H., 1988. Stochastic modeling of seafloor morphology: Inversion of Sea Beam data for second-order statistics. Journal of Geophysical Research B11, 13589-13608.

Grams, P.E., Topping, D.J., Schmidt, J.C., Hazel, J.E., Kaplinski, M., 2013. Linking morphodynamic response with sediment mass balance on the Colorado River in Marble Canyon: Issues of scale, geomorphic setting, and sampling design. Journal of Geophysical Research - Earth Surface 118, $361-381$.

Guadagnini, A., Neuman, S.P., 2011. Extended power-law scaling of self-affine signals exhibiting apparent multifractality. Geophysical Research Letters 38. doi:10.1029/2011GL047727.

Hani, A.F.M., Sathyamoorthy, D., Asirvadam, V.S., 2011. A method for computation of surface roughness of digital elevation model terrains via multiscale analysis. Computers and Geosciences 37, $177-$ 192. doi:http://dx.doi.org/10.1016/j.cageo.2010.05.021.

Hilldale, R.C., Raff, D., 2008. Assessing the ability of airborne LiDAR to map river bathymetry. Earth Surface Processes and Landforms 33, 773-783. doi:10.1002/esp. 1575.

Hodge, R., Brasington, J., Richards, K., 2009. In situ characterization of grain-scale fluvial morphology using terrestrial laser scanning. Earth Surface Processes and Landforms 34, 954-968.

Hough, S.E., 1989. On the use of spectral methods for the determination of fractal dimension. Geophysical Research Letters 16, 673-676.

Huang, J., Turcotte, D.L., 1990. Fractal image analysis: application to the topography of oregon and synthetic images. Journal of the Optical Society of America A 7, 1124-1130.

Huber, P.J., 1981. Robust Statistics. John Wiley and Sons, Inc., New York.

Hunter, J.D., 2007. Matplotlib: A 2D graphics environment. Computing in Science and Engineering 9, 90-95. doi:10.1109/MCSE. 2007.55.

Jackson, D.R., Richardson, M.D., 2007. High-frequency seafloor acoustics. Springer, New York.

James, M.R., Robson, S., 2012. Straightforward reconstruction of 3D surfaces and topography with a camera: Accuracy and geoscience application. Journal of Geophysical Research - Earth Surface 117, 
F03017. doi:10.1029/2011JF002289

Jerolmack, D.J., Paola, C., 2010. Shredding of environmental signals by sediment transport. Geophysical Research Letters 37.

Jones, E., Oliphant, T., Peterson, P., et al., 2001-. SciPy: Open source scientific tools for Python. URL: http://www.scipy.org/. [Online; accessed 2015-02-15].

Kalbermatten, M., Ville, D.V.D., Turberg, P., Tuia, D., Joost, S., 2012. Multiscale analysis of geomorphological and geological features in high resolution digital elevation models using the wavelet transform. Geomorphology 138, 352 - 363. doi:http://dx.doi.org/10.1016/j.geomorph.2011.09.023.

Kaplinski, M., Hazel, J.E., Grams, P.E., Davis, P.A., 2014. Monitoring fine-sediment volume in the Colorado River ecosystem, Arizona: Construction and analysis of digital elevation models. U.S. Geological Survey Open-File Report 20141052, 29 p.

Kaplinski, M., Hazel, J.E., Parnell, R., Breedlove, M., Kohl, K., Gonzales, M., 2009. Monitoring finesediment volume in the Colorado River Ecosystem, Arizona: Bathymetric survey techniques. U.S. Geological Survey Open-file Report 2009-1207, 41 pp.

von Karman, T., Howarth, L., 1938. On the statistical theory of isotropic turbulence. Proceedings of the Royal Society London Series A 164, 192-215.

Keller, J.M., Crownover, R.M., Chen, R.Y., 1987. Characteristics of natural scenes related to the fractal dimension. IEEE Transactions on Pattern Analysis and Machine Intelligence 9, 621-627.

Knuth, D.E., 1998. The Art of Computer Programming, volume 2: Semi - numerical Algorithms. 3rd ed., Addison-Wesley, Boston.

Krieger, L., Peacock, J.R., 2014. MTpy: A Python toolbox for magnetotellurics. Computers and Geosciences 72, 167-175.

Kukko, A., Anttila, K., Manninen, T., Kaasalainen, S., Kaartinen, H., 2013. Snow surface roughness from mobile laser scanning data. Cold Regions Science and Technology 96, 23-35.

Lashermes, B., Foufoula-Georgiou, E., Dietrich, W.E., 2007. Channel network extraction from high resolution topography using wavelets. Geophysical Research Letters 34, L23S04.

Lassueur, T., Joost, S., Randin, C., 2006. Very high resolution digital elevation models: do they improve models of plant species distribution? Ecological Modelling 198, 139-153.

Legleiter, C.J., Overstreet, B.T., 2012. Mapping gravel-bed river bathymetry from space. Journal of Geophysical Research - Earth Surface 117, F04024.

Longuet-Higgins, M.S., 1975. On the joint distribution of the periods and amplitudes of sea waves. Journal of Geophysical Research 80, 2688-2694.

Mallet, C., Bretar, F., 2009. Full waveform topographic LiDAR: State-of-the-art. ISPRS Journal of Photogrammetry and Remote Sensing 64, 1-16.

Maneewongvatana, S., Mount, D.M., 1999. It's okay to be skinny, if your friends are fat, in: 4th Annual CGC Workshop on Computational Geometry, Center for Geometric Computing. URL: http: //www.cs.umd.edu/ mount/Papers/cgc99-smpack.pdf.

Manes, C., Guala, M., Löwe, H., Bartlett, S., Egli, L., Lehning, M., 2008. Statistical properties of fresh snow roughness. Water Resources Research 44, W11407.

Mayer, L.A., 2006. Frontiers in seafloor mapping and visualization. Marine Geophysical Researches 27, $7-17$.

Mazzarini, F., Pareschi, M.T., Favalli, M., Isola, I., Tarquini, S., Boschi, E., 2007. Lava flow identification and aging by means of LiDAR intensity: Mount Etna case. Journal of Geophysical Research - Solid Earth 112, B02201.

Miller, L., Parsons, C., 1990. Rough surface scattering results based on bandpass autocorrelation forms. IEEE Transactions on Geoscience and Remote Sensing 28, 1017-1021.

Millman, K.J., Aivazis, M., 2011. Python for scientists and engineers. Computing in Science and Engineering 13, 9-12. doi:10.1109/MCSE. 2011.36.

Nelson, P.A., Bellugi, D., Dietrich, W.E., 2014. Delineation of river bed-surface patches by clustering high-resolution spatial grain size data. Geomorphology 205, 102-119.

Nield, J.M., Wiggs, G.F.S., Squirrell, R.S., 2011. Aeolian sand strip mobility and protodune development on a drying beach: examining surface moisture and surface roughness patterns measured by terrestrial laser scanning. Earth Surface Processes and Landforms 36, 513-522.

Nikora, V., 2005. High-order structure functions for planet surfaces: A turbulence metaphor. IEEE Geoscience and Remote Sensing Letters 2, 362-365.

Nikora, V.I., Goring, D., Biggs, B.J.F., 1998. On gravel-bed roughness characterisation. Water Resources Research 34, 517-527.

Nitsche, M., Turowski, J.M., Badoux, A., Rickenmann, D., Kohoutek, T.K., Pauli, M., Kirchner, J.W., 2013. Range imaging: a new method for high-resolution topographic measurements in small- and 
medium-scale field sites. Earth Surface Processes and Landforms 38, 810-825. doi:10.1002/esp.3322.

Oliphant, T.E., 2007. Python for scientific computing. Computing in Science and Engineering 9, 10-20. doi:10.1109/MCSE. 2007.58

Oppermann, N., Selig, M., Bell, M.R., Enßlin, T.A., 2013. Reconstruction of Gaussian and log-normal fields with spectral smoothness. Physical Review E 112, 032136.

Parsons, D.R., Best, J.L., Orfeo, O., Hardy, R.J., Kostaschuk, R., Lane, S.N., 2005. Morphology and flow fields of three-dimensional dunes, Rio Parana, Argentina: Results from simultaneous multibeam echo sounding and acoustic Doppler current profiling. Journal of Geophysical Research - Earth Surface 110, F04S03.

Passalacqua, P., Tarolli, P., Foufoula-Georgiou, E., 2010. Testing space-scale methodologies for automatic geomorphic feature extraction from LiDAR in a complex mountainous landscape. Water Resources Research 46, W11535.

Pelgrum, H., Schmugge, T., Rango, A., Ritchie, J., Kustas, B., 2000. Length-scale analysis of surface albedo, temperature, and normalized difference vegetation index in desert grassland. Water Resources Research 36, 1757-1765.

Perron, J.T., Kirchner, J.W., Dietrich, W.E., 2008. Spectral signatures of characteristic spatial scales and nonfractal structure in landscapes. Journal of Geophysical Research - Earth Surface 113, F04003.

Pike, R., Wesley, J., 1975. Spectral analysis of landforms. Annals of the Association of American Geographers 65, 499-516.

Pirotti, F., Tarolli, P., 2010. Suitability of LiDAR point density and derived landform curvature maps for channel network extraction. Hydrological Processes 24, 1187-1197. doi:10.1002/hyp.7582.

Pollyea, R.M., Fairley, J.P., 2011. Estimating surface roughness of terrestrial laser scan data using orthogonal distance regression. Geology 39, 623-626.

Pollyea, R.M., Fairley, J.P., 2012. Experimental evaluation of terrestrial LiDAR-based surface roughness estimates. Geosphere 8, 222-228.

Pradervand, J.N., Dubuis, A., Pellissier, L., Guisan, A., Randin, C., 2014. Very high resolution environmental predictors in species distribution models: Moving beyond topography? Progress in Physical Geography 38, 79-96. doi:10.1177/0309133313512667.

Press, W.H., Teukolsky, S.A., Vetterling, W.T., Flannery, B.P., 2007. Numerical Recipes 3rd Edition: The Art of Scientific Computing. Cambridge University Press. doi:ISBN-13:9780521880688.

Priestley, M.B., 1981. Spectral analysis and time-series. Academic Press, New York.

Ramachandran, P., Varoquaux, G., 2011. Mayavi: 3D Visualization of Scientific Data. Computing in Science and Engineering 13, 40-51.

Roering, J.J., Mackey, B.H., Marshall, J.A., Sweeney, K.E., Deligne, N.I., Booth, A.M., Handwerger, A.L., Cerovski-Darriau, C., 2013. You are HERE: Connecting the dots with airborne LiDAR for geomorphic fieldwork. Geomorphology 200, 172-183.

Rothrock, D., Thorndike, A., 1980. Geometric properties of the underside of sea ice. Journal of Geophysical Research: Oceans 85, 3955-3963.

Rozema, W., 1968. The use of spectral analysis in describing lunar surface roughness. U.S. Geological Survey Professional Paper 650-D.

Rushing, J., Ramachandrana, R., Nairb, U., Graves, S., Welch, R., Lin, H., 2005. ADaM: A data mining toolkit for scientists and engineers. Computers and Geosciences 31, 607-618.

Rychkov, I., Brasington, J., Vericat, D., 2012. Computational and methodological aspects of terrestrial surface analysis based on point clouds. Computers and Geosciences 42, 64-70.

Sankey, J.B., Glenn, N.F., Germino, M.J., Gironella, A.I.N., Thackray, G.D., 2010. Relationships of aeolian erosion and deposition with LiDAR-derived landscape surface roughness following wildfire. Geomorphology 119, 135-145.

Savitzky, A., Golay, M.J.E., 1964. Smoothing and differentiation of data by simplified least squares procedures. Analytical Chemistry 36, 1627-1639.

Sayles, R.S., Thomas, T.R., 1978. Surface topography as a nonstationary random process. Nature 271, 431-434.

Selig, M., Bell, M.R., Junklewitz, H., Oppermann, N., Reinecke, M., Greiner, M., Pachajoa, C., Enßlin, T.A., 2013. NIFTY - Numerical information field theory - a versatile Python library for signal inference. Astronomy and Astrophysics 554, A26.

Shepard, M.K., Brackett, R.A., Arvidson, R.E., 1995. Self-affine (fractal) topography: Surface parameterization and radar scattering. Journal of Geophysical Research: Planets 100, 11709-11718. doi:10.1029/95JE00664.

Shepard, M.K., Campbell, B.A., Bulmer, M.H., Farr, T.G., Gaddis, L.R., Plaut, J.J., 2001. The roughness of natural terrain: A planetary and remote sensing perspective. Journal of Geophysical Research: 
Planets 106, 32777-32795. doi:10.1029/2000JE001429.

Singh, A., Foufoula-Georgiou, E., Port-Agel, F., Wilcock, P.R., 2012. Coupled dynamics of the coevolution of gravel bed topography, flow turbulence and sediment transport in an experimental channel. Journal of Geophysical Research - Earth Surface 117, F04016.

Smith, M.W., 2014. Roughness in the Earth Sciences. Earth-Science Reviews 136, 202-225.

Tarolli, P., 2014. High-resolution topography for understanding Earth surface processes: Opportunities and challenges. Geomorphology 216, 295-312.

Taylor, G.I., 1938. The spectrum of turbulence. Proceedings of the Royal Society London Series A 164, 476-490.

Trevisani, S., Cavalli, M., Marchi, L., 2012. Surface texture analysis of a high-resolution DTM: Interpreting an alpine basin. Geomorphology 161162, 26 - 39. doi:http://dx.doi.org/10.1016/j.geomorph. 2012.03 .031

Turcotte, D., 1992. Fractals and Chaos in Geology and Geophysics. Cambridge Univ. Press, Cambridge. Vierling, K.T., Vierling, L.A., Gould, W.A., Martinuzzi, S., Clawges, R.M., 2008. LiDAR: shedding new light on habitat characterization and modeling. Frontiers in Ecology and the Environment 6, 90-98. van der Walt, S., Colbert, S.C., Varoquaux, G., 2011. The NumPy array: A structure for efficient numerical computation. Computing in Science and Engineering 13, 22-30. doi:10.1109/MCSE. 2011.37.

Warrick, J.A., Rubin, D.M., Ruggiero, P., Harney, J., Draut, A.E., Buscombe, D., 2009. Cobble cam: grain-size measurements of sand to boulder from digital photographs and autocorrelation analyses. Earth Surface Processes and Landforms 34, 1811-1821.

Welford, B.P., 1962. Note on a method for calculating corrected sums of squares and products. Technometrics 4, 419-420.

Wellmann, J.F., Croucher, A., Regenauer-Lieb, K., 2012. Python scripting libraries for subsurface fluid and heat flow simulations with TOUGH2 and SHEMAT. Computers and Geosciences 43, $197-206$.

Westoby, M., Brasington, J., Glasser, N., Hambrey, M., Reynolds, J., 2012. Structure-from-motion photogrammetry: A low-cost, effective tool for geoscience applications. Geomorphology 179, $300-314$.

Wheaton, J.M., Brasington, J., Darby, S.E., Merz, J., Pasternack, G.B., Sear, D., Vericat, D., 2010. Linking geomorphic changes to salmonid habitat at a scale relevant to fish. River Research and Applications 26, 469-486. doi:10.1002/rra.1305.

Whitehouse, D., 1997. Surface metrology. Measurement Science and Technology 8, 955-972.

Wieland, R., Dalchow, C., 2009. Detecting landscape forms using Fourier transformation and singular value decomposition(SVD). Computers and Geosciences 35, 1409-1414.

Wilson, T.H., Dominic, J., 1998. Fractal inter-relationships between topography and structure. Earth Surface Processes and Landforms 23, 509-525.

Woodget, A., Carbonneau, P., Visser, F., Maddock, I., 2015. Quantifying submerged fluvial topography using hyperspatial resolution uas imagery and structure from motion photogrammetry. Earth Surface Processes and Landforms 40, 47-64.

Wright, S.A., Kaplinski, M., 2011. Flow structures and sandbar dynamics in a canyon river during a controlled flood, Colorado River, Arizona. Journal of Geophysical Research - Earth Surface 116, F01019.

van Zyl, J., Burnette, C., Farr, T., 1991. Inference of surface power spectra from inversion of multifrequency polarimetric radar data. Geophysical Research Letters 18, 1787-1790. 
Table captions

(1) PySESA sub-modules (to date) and their functions.

\section{Figure captions}

(1) A schematic of a basic PySESA workflow (read left to right) and the sub-modules responsible for carrying out tasks.

(2) Illustration of the data windowing procedure controlled by the PySESA: : partition parameter 'percent overlap'. A dense point cloud is analysed such that is decimated to a regular $1 \mathrm{~m} \times 1 \mathrm{~m}$ grid (red dots) by using increasing amounts of data: a) $-50 \%$ overlap; b) $0 \%$ overlap (program default); c) 50\% overlap; and d) 100\% overlap.

(3) Each of the 4 subplots shows the same point cloud (red dots) in a small area typical of a window of data, and the $2 \mathrm{D}$ function fit through that point cloud (blue surface) for the purposes of detrending. The 4 detrending methods currently implemented in PySESA are a) Ordinary Least Squares (OLR); b) Robust Linear Regression (RLR); c) Orthogonal Distance Regression (ODR); and d) Savitsky-Golay digital filter of any order (shown is order 0 ). The detrending effects on the point cloud are shown by the standard deviation of detrended amplitudes, denoted $\sigma$ in each subplot, and which range from $6.1 \mathrm{~cm}$ (ODR) to $29.1 \mathrm{~cm}$ (RLR).

(4) The distribution of residuals created by detrending the point clouds in the corresponding 4 subplots of Figure 3.

(5) a) The raw point cloud used to demonstrate the functionality of the PySESA toolbox. This is a bathymetric point cloud, obtained using multibeam echosounder, of a 60 $\times 80 \mathrm{~m}$ patch of the Colorado River bed in Western Grand Canyon, around river mile 224. The point cloud, composed of almost 1 million 3D points, clearly shows areas of varying textures, including sand dunes, flat sand areas, and rocky areas. b) A different perspective on the same scene, to better show the variation in heights across the data.

(6) The point cloud shown in Figure 5a, decimated to a $0.25 \times 0.25 \mathrm{~m}$ regular grid by the PySESA program, and colour-coded by: a) spectral root-mean-square variation in amplitude, $\sigma(\mathrm{m})$; and b) spectral strength $\omega_{2}\left(\mathrm{~m}^{4}\right)$.

(7) Contour maps of gridded $(0.25 \times 0.25 \mathrm{~m})$ parameters from spatial and spectral analyses of the point cloud shown in Figure 5. In each subplot, just a small $70 \times 45 \mathrm{~m}$ portion of the data is shown. The parameters shown are: a) elevation; b) spectral strength; c) spectral slope; d) integral lengthscale; e) spectral width; f) standard deviation; g) detrended standard deviation; h) spectral standard deviation; i) ratio of integral lengthscale and standard deviation; and j) skewness.

(8) Processing times for increasing numbers of $3 \mathrm{D}$ points in the point cloud, for processing for a a) 4-core Intel ${ }^{\circledR}$ Xeon ${ }^{\circledR}$ W3530 CPU at 2.80GHz; and a b) 8-core Intel ${ }^{\circledR}$ Core ${ }^{\circledR}$ i7-3630QM CPU at $2.40 \mathrm{GHz}$. The overall differences in the processing times show how distributing the computation over more CPUs (b) is more beneficial than a faster CPU (a). Different symbols refer to the degree of overlap in the windowing procedure. Connected symbols show processing times all spatial parameters using PySESA: : spatial and unconnected symbols show processing times all spatial and spectral parameters using PySESA: :spectral. 
1016

1017

1018

1019

(9) The percentage speedup associated with processing with an 8-core $2.40 \mathrm{GHz}$ compared with a 4-core $2.80 \mathrm{Ghz}$ processor, for a) all spatial and spectral parameters using PySESA : : spectral, and b) processing all spatial parameters using PySESA : : spatial. Different symbols refer to the degree of overlap in the windowing procedure. 


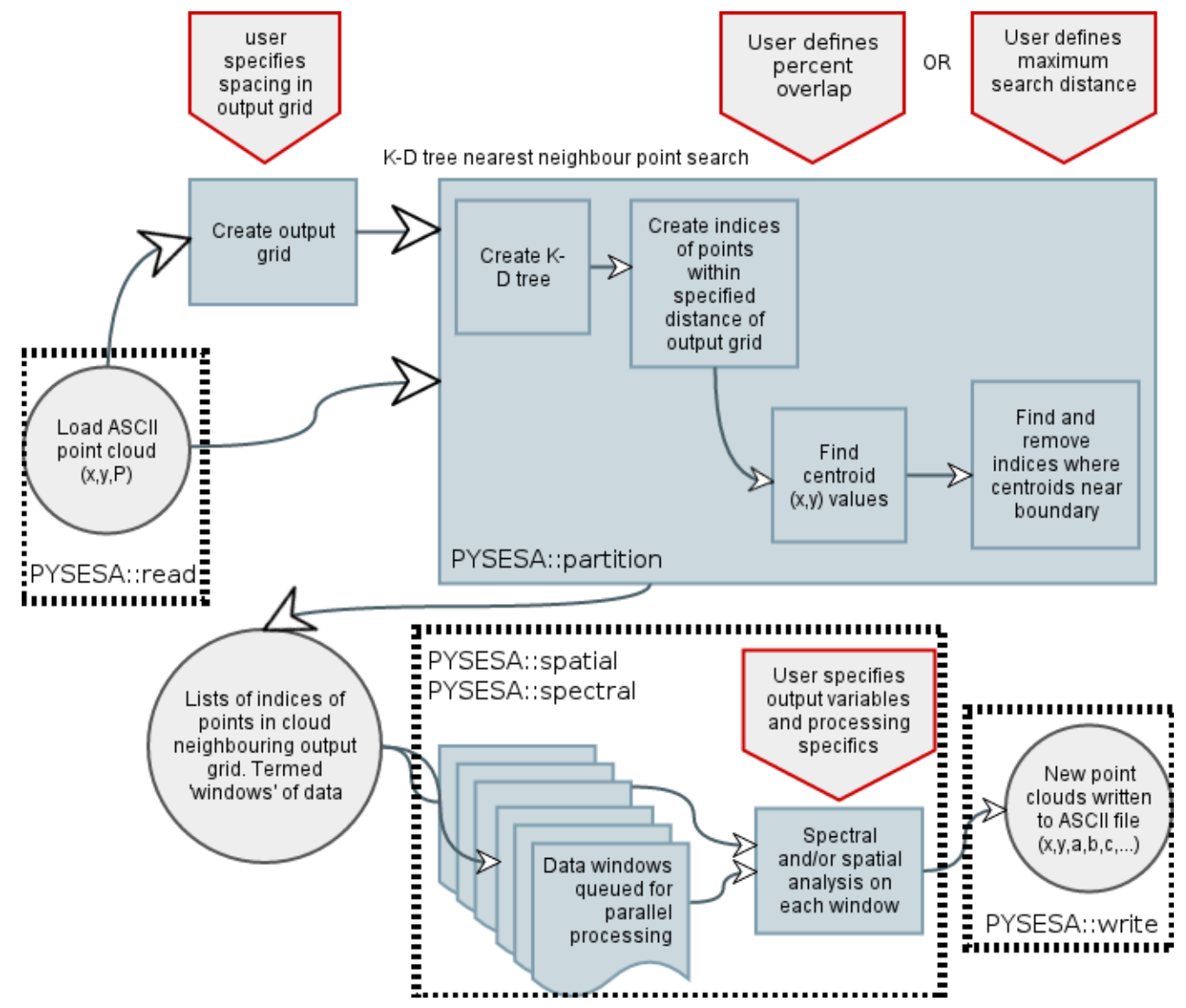

Figure 1: A schematic of a basic PySESA workflow (read left to right) and the sub-modules responsible for carrying out tasks. 

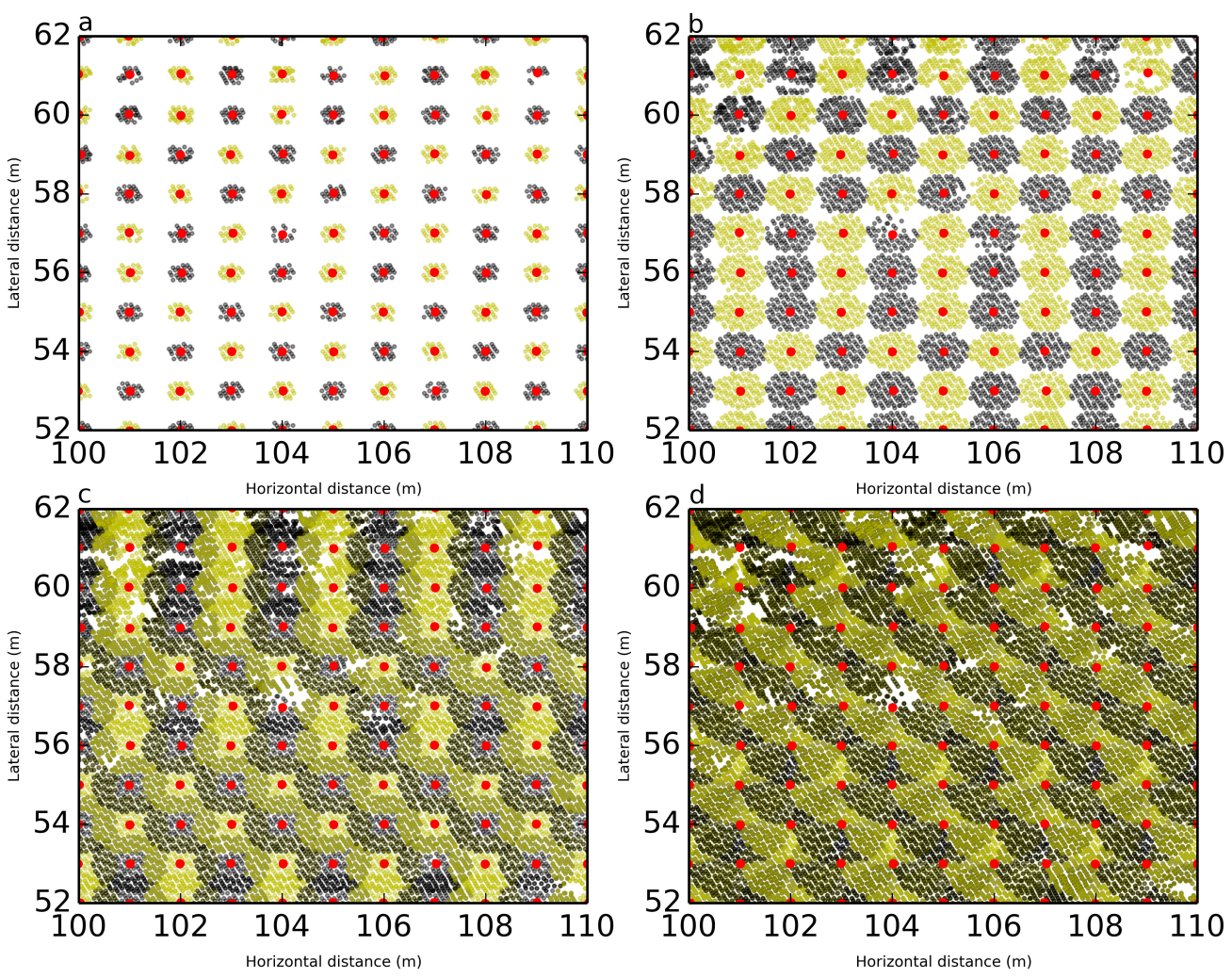

Figure 2: Illustration of the data windowing procedure controlled by the PySESA: partition parameter 'percent overlap'. A dense point cloud is analysed such that is decimated to a regular $1 \mathrm{~m} \times 1 \mathrm{~m}$ grid (red dots) by using increasing amounts of data: a) $-50 \%$ overlap; b) $0 \%$ overlap (program default); c) $50 \%$ overlap; and d) $100 \%$ overlap. 

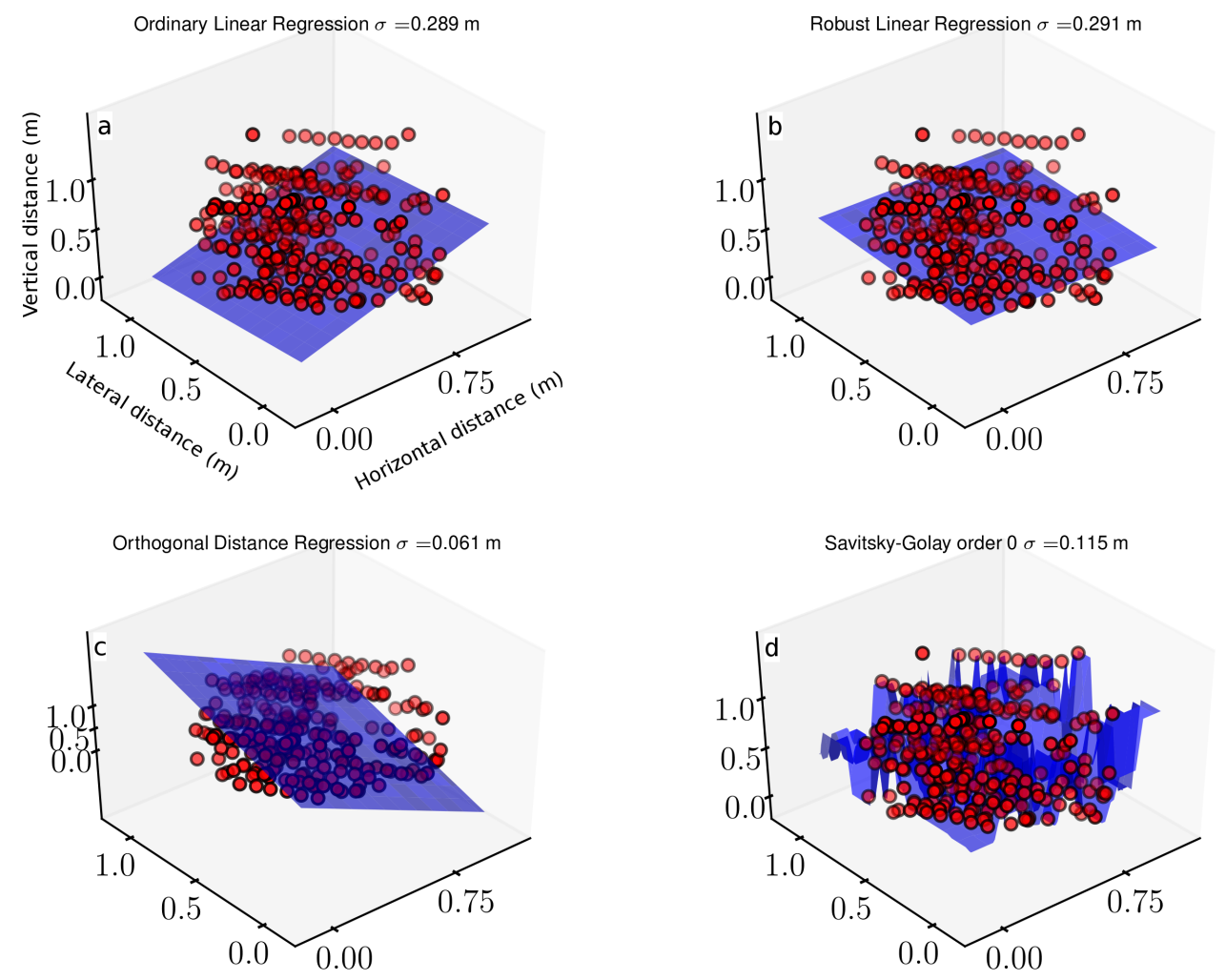

Figure 3: Each of the 4 subplots shows the same point cloud (red dots) in a small area typical of a window of data, and the $2 \mathrm{D}$ function fit through that point cloud (blue surface) for the purposes of detrending. The chosen point cloud shows a high degree of clustering in space, which means that the 4 detrending methods currently implemented in PySESA give very different trends through the data. These method choices are a) Ordinary Least Squares (OLR); b) Robust Linear Regression (RLR); c) Orthogonal Distance Regression (ODR); and d) Savitsky-Golay digital filter of any order (shown is order 0 ). The detrending effects on the point cloud are shown by the standard deviation of detrended amplitudes, denoted $\sigma$ in each subplot, and which range from $6.1 \mathrm{~cm}(\mathrm{ODR})$ to $29.1 \mathrm{~cm}$ (RLR). 

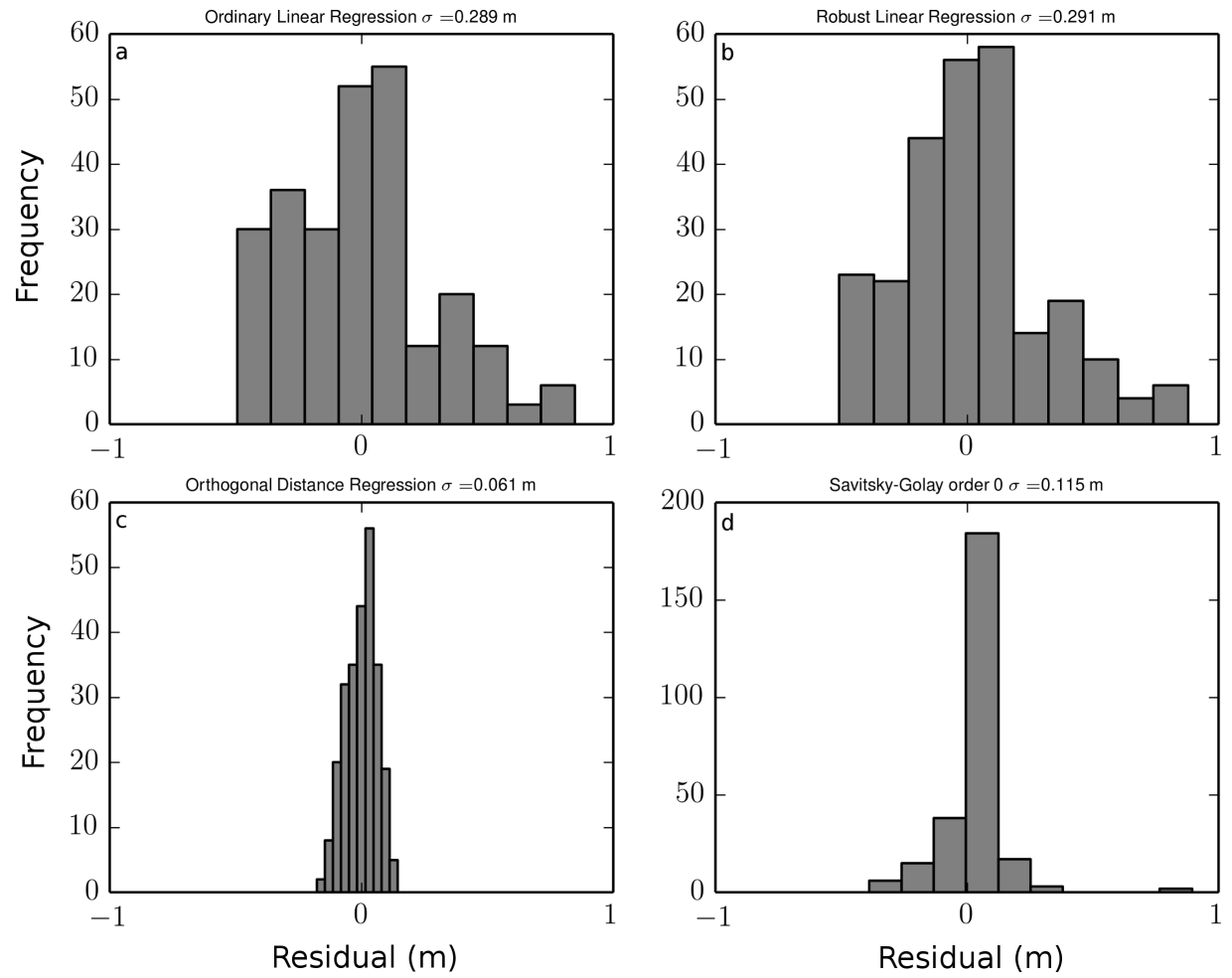

Figure 4: The distribution of residuals created by detrending the point clouds in the corresponding 4 subplots of Figure 3. 

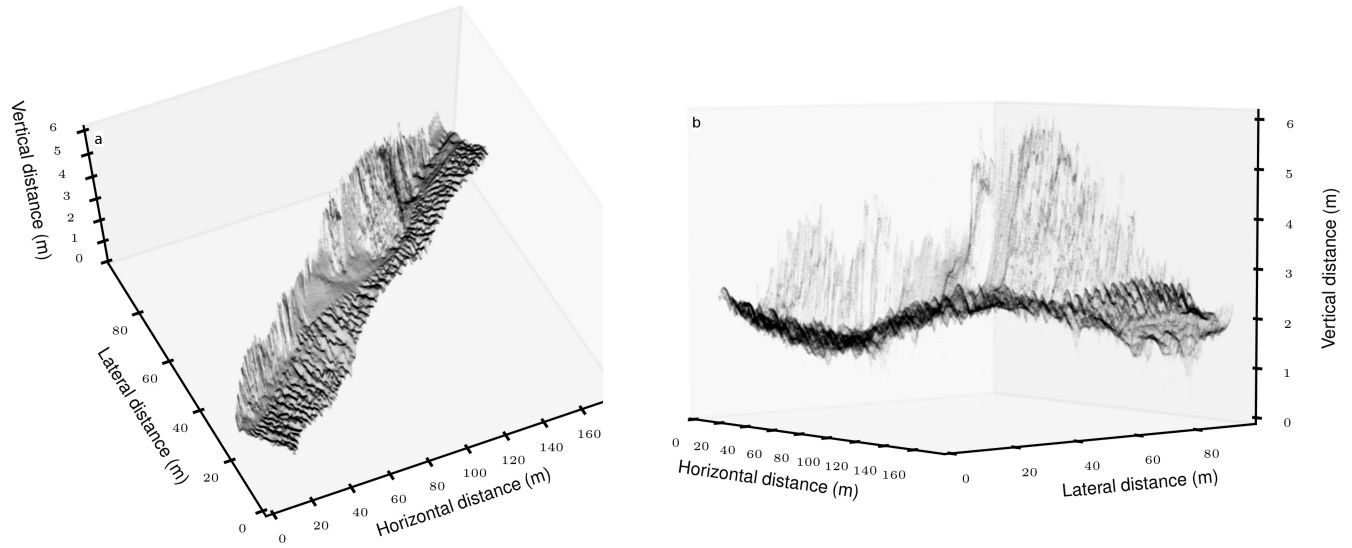

Figure 5: a) The raw point cloud used to demonstrate the functionality of the PySESA toolbox. This is a bathymetric point cloud, obtained using multibeam echosounder, of a $60 \times 80 \mathrm{~m}$ patch of the Colorado River bed in Western Grand Canyon, around river mile 224. The point cloud, composed of almost 1 million 3D points, clearly shows areas of varying textures, including sand dunes, flat sand areas, and rocky areas. b) A different perspective on the same scene, to better show the variation in heights across the data.
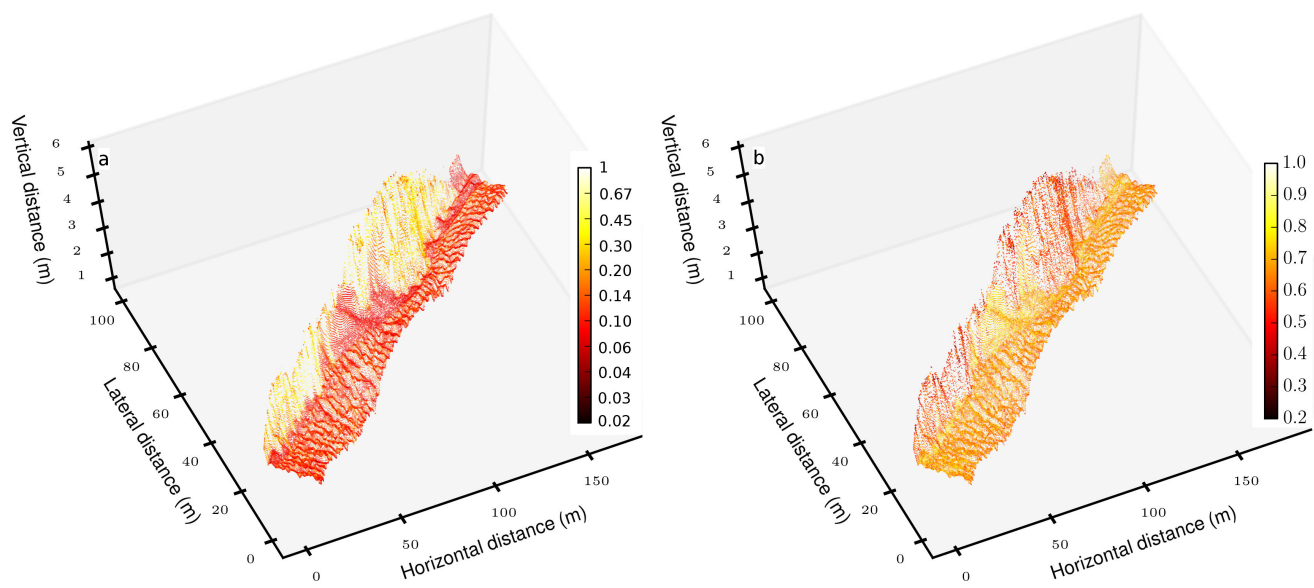

Figure 6: The point cloud shown in Figure 5a, decimated to a $0.25 \times 0.25 \mathrm{~m}$ regular grid by the PySESA program, and colour-coded by: a) spectral root-mean-square variation in amplitude, $\sigma$ $(\mathrm{m})$; and b) spectral strength $\omega_{2}\left(\mathrm{~m}^{4}\right)$. 


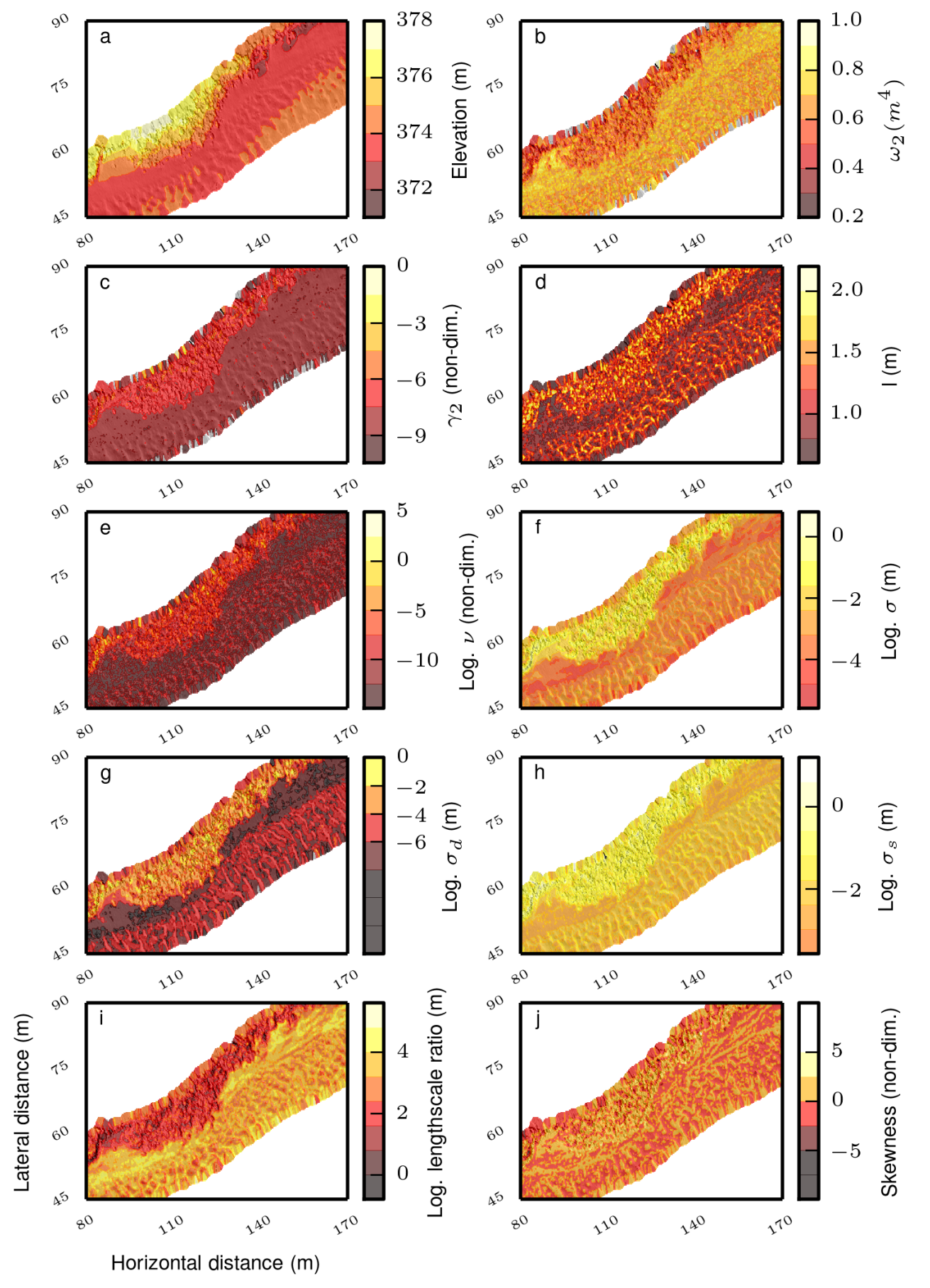

Figure 7: Contour maps of gridded $(0.25 \times 0.25 \mathrm{~m})$ parameters from spatial and spectral analyses of the point cloud shown in Figure 5. In each subplot, just a small $70 \times 45 \mathrm{~m}$ portion of the data is shown. The parameters shown are: a) elevation; b) spectral strength; c) spectral slope; d) integral lengthscale; e) spectral width; f) standard deviation; g) detrended standard deviation; h) spectral standard deviation; i) ratio of integral lengthscale and standard deviation; and j) skewness. 

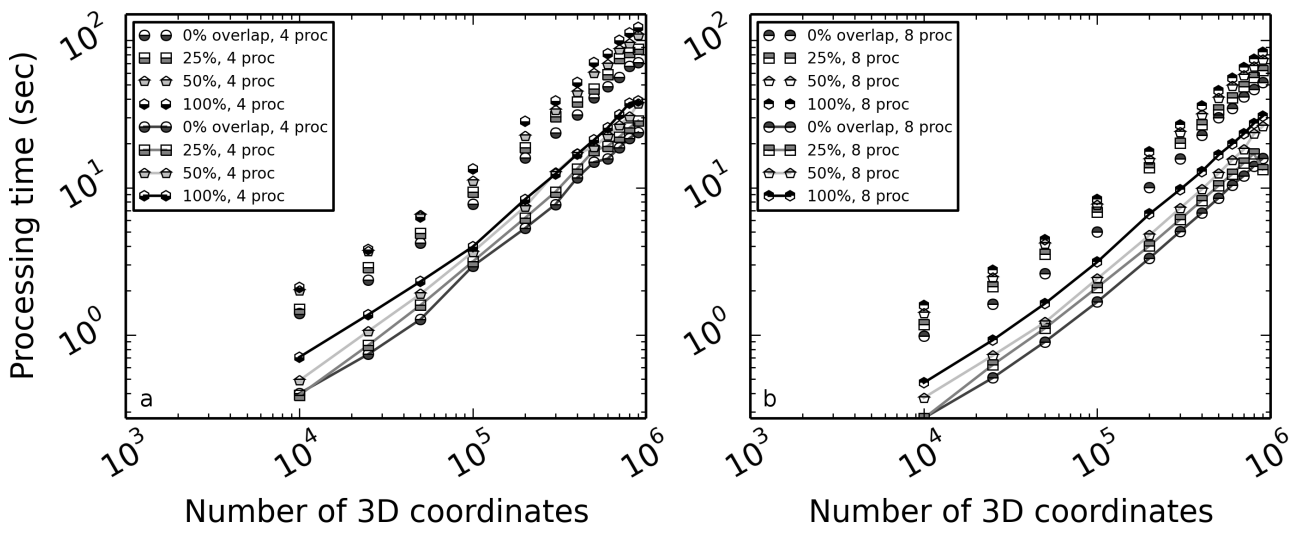

Figure 8: Processing times for increasing numbers of 3D points in the point cloud, for processing for a a) 4-core Intel ${ }^{\circledR}$ Xeon ${ }^{\circledR}$ W3530 CPU at $2.80 \mathrm{GHz}$; and a b) 8-core Intel ${ }^{\circledR}$ Core ${ }^{\circledR}$ i7-3630QM $\mathrm{CPU}$ at $2.40 \mathrm{GHz}$. The overall differences in the processing times show how distributing the computation over more CPUs (b) is more beneficial than a faster CPU (a). Different symbols refer to the degree of overlap in the windowing procedure. Connected symbols show processing times all spatial parameters using PySESA: : spatial and unconnected symbols show processing times all spatial and spectral parameters using PySESA : spectral.
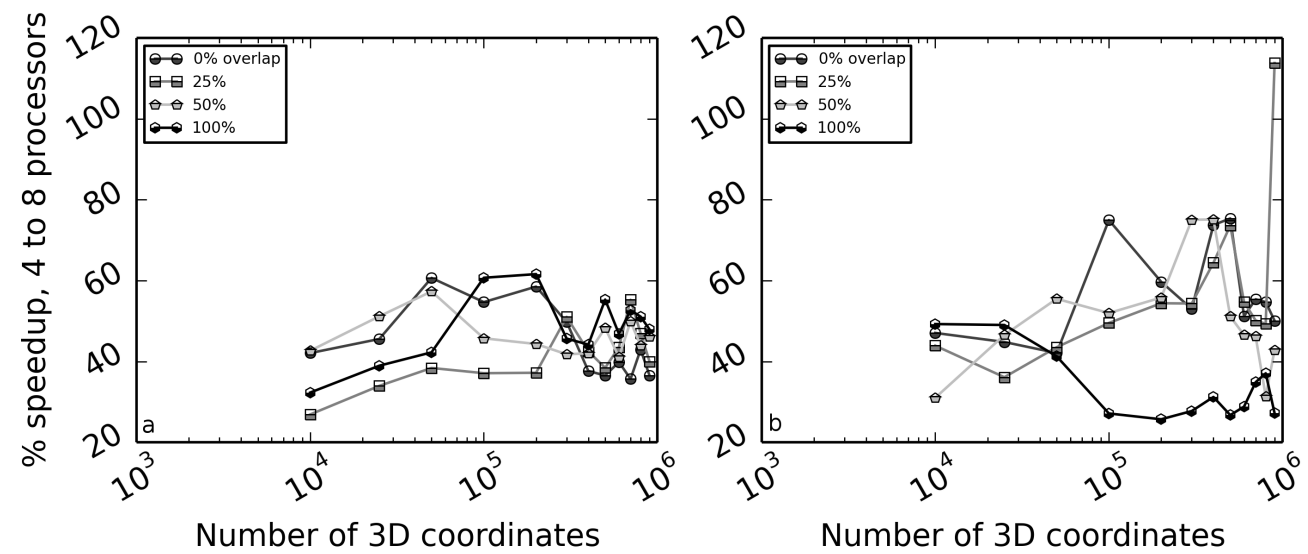

Figure 9: The percentage speedup associated with processing with an 8-core $2.40 \mathrm{GHz}$ compared with a 4-core $2.80 \mathrm{Ghz}$ processor, for a) all spatial and spectral parameters using PySESA: :spectral, and b) processing all spatial parameters using PySESA: spatial. Different symbols refer to the degree of overlap in the windowing procedure. 
Table 1: PySESA sub-modules (to date) and their functions.

\begin{tabular}{|c|c|}
\hline PySESA sub-module & Function \\
\hline read & read a 3-column space, comma or tab delimited text file \\
\hline partition & $\begin{array}{l}\text { partition a } N \times 3 \text { point cloud }(P=[X, Y, Z]) \text { into } m \text { windows of } n \times 3 \text { points } \\
\left(\mathbb{P}_{m}\right) \text { with specified spacing between centroids of adjacent windows } \\
\text { and with specified overlap between windows. }\end{array}$ \\
\hline detrend & .getdata() returns detrended amplitudes of a $N \times 3$ point cloud \\
\hline sgolay & - getdata() returns the Savitsky-Golay digital filter of a 2D signal \\
\hline spatial & $\begin{array}{l}\text { calculate spatial statistics of a Nx3 point cloud } \\
\text { ·getdata() returns: } \\
\mathrm{x}=\text { centroid in horizontal coordinate } \\
\mathrm{y}=\text { centroid in laterial coordinate } \\
\mathrm{z} \text { _mean }=\text { centroid in amplitude } \\
\text { z_max }=\text { max amplitude } \\
\text { z_min }=\text { min amplitude } \\
\text { z_range }=\text { range in amplitude } \\
\text { sigma }\left(\sigma \text { or } \sigma_{d}, \text { unit amplitude) }=\text { standard deviation of amplitudes }\right. \\
\text { skewness (non-dim.) = skewness of amplitudes } \\
\text { kurtosis (non-dim.) }=\text { skewness of amplitudes } \\
\mathrm{n}=\text { number of } 3 \mathrm{D} \text { coordinates }\end{array}$ \\
\hline RunningStats & called by spatial to compute sigma, skewness and kurtosis \\
\hline lengthscale & calculates the integral lengthscale of a $N \times 3$ point cloud \\
\hline spectral & 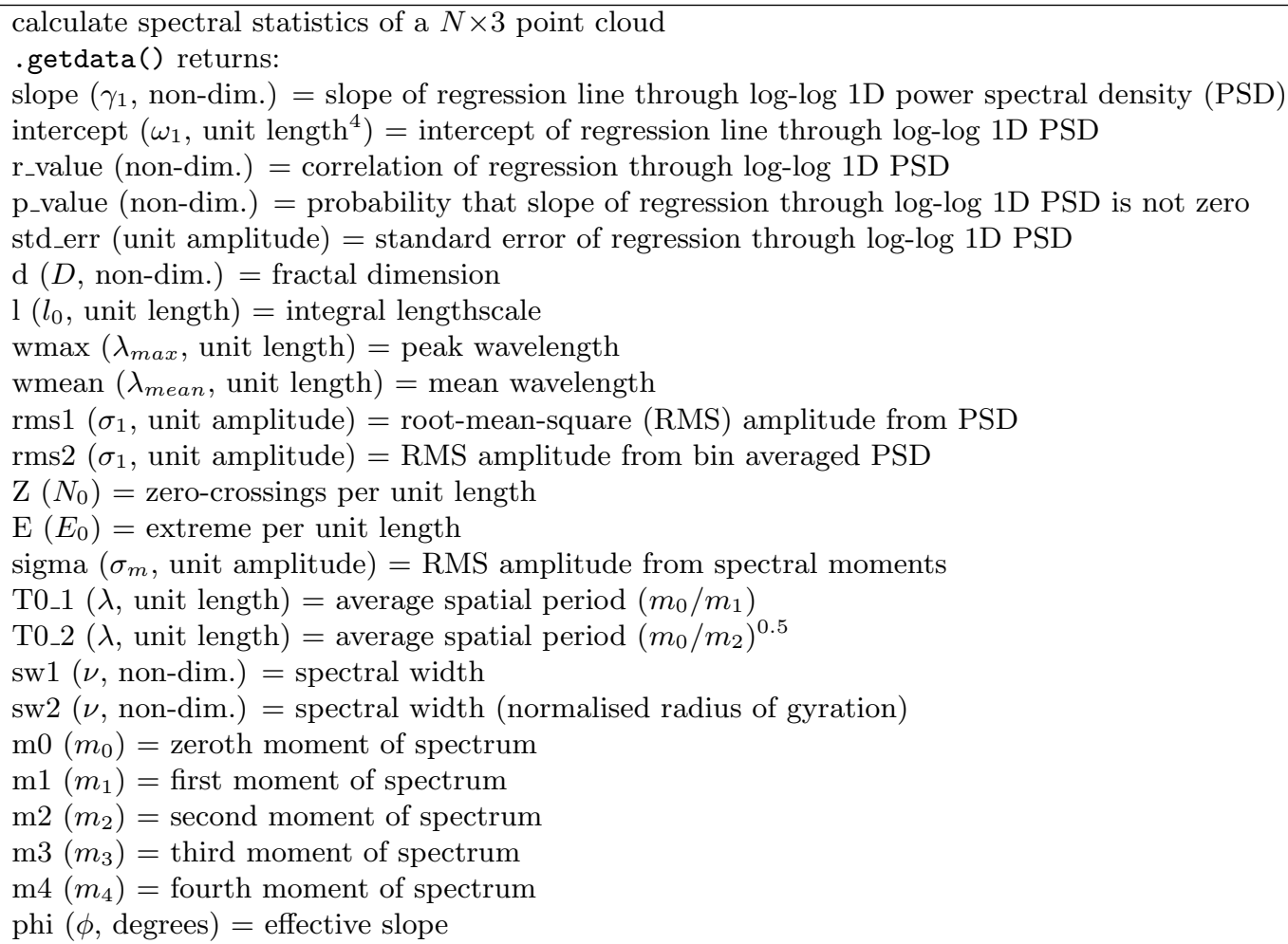 \\
\hline process & allows control of inputs to all modules (full workflow) \\
\hline write & write program outputs to a comma delimited text file \\
\hline plot & various utilities for plosting raw and decimated point clouds and grids in 2D and 3D \\
\hline test & program testing suite \\
\hline
\end{tabular}

